\title{
CHEMICAL COMPOSITIONS OF THIN-DISK, HIGH-METALLICITY RED HORIZONTAL-BRANCH FIELD STARS
}

\author{
M. AfŞAR ${ }^{1,2}$, C. SNEDEN ${ }^{2}$, AND B.-Q. For ${ }^{2,3}$ \\ ${ }^{1}$ Department of Astronomy and Space Sciences, Ege University, 35100 Bornova, İzmir, Turkey; melike.afsar@ege.edu.tr \\ ${ }^{2}$ Department of Astronomy and McDonald Observatory, The University of Texas, Austin, TX 78712, USA; \\ afsar@astro.as.utexas.edu, chris@astro.as.utexas.edu, biqing@ astro.as.utexas.edu \\ ${ }^{3}$ ICRAR, University of Western Australia, 35 Stirling Hwy, Crawley, WA 6009, Australia; biqing.for@uwa.edu.au \\ Received 2012 March 19; accepted 2012 May 15; published 2012 June 11
}

\begin{abstract}
We present a detailed abundance analysis and atmospheric parameters of 76 stars from a survey to identify field Galactic red horizontal-branch (RHB) stars. High-resolution echelle spectra $(R \simeq 60,000, \mathrm{~S} / \mathrm{N} \geqslant 100)$ were obtained with the $2.7 \mathrm{~m}$ Harlan J. Smith Telescope at McDonald Observatory. The target stars were selected only by color and parallax information. Overall metallicities and relative abundances of proton-capture elements $(\mathrm{C}, \mathrm{N}, \mathrm{O}, \mathrm{Li})$, $\alpha$-elements ( $\mathrm{Ca}$ and $\mathrm{Si}$ ), and neutron-capture elements (Eu and $\mathrm{La}$ ) were determined by either equivalent width or synthetic spectrum analyses. We used $\mathrm{CN}$ features at the $\lambda \lambda 7995-8040$ region in order to determine the ${ }^{12} \mathrm{C} /{ }^{13} \mathrm{C}$ ratios of our targets. Investigation of the evolutionary stages, using spectroscopic $T_{\text {eff }}$ and $\log g$ values along with derived ${ }^{12} \mathrm{C} /{ }^{13} \mathrm{C}$ ratios, revealed the presence of 18 probable RHB stars in our sample. We also derived kinematics of the stars with available distance information. Taking into account both the kinematics and probable evolutionary stages, we conclude that our sample contains 5 thick-disk and 13 thin-disk RHB stars. Up until now, RHB stars have been considered as members of the thick disk, and were expected to have large space velocities and sub-solar metallicities. However, our sample is dominated by low-velocity solar-metallicity RHB stars; their existence cannot be easily explained with standard stellar evolution.
\end{abstract}

Key words: Galaxy: evolution - stars: abundances - stars: evolution - stars: kinematics and dynamics

Online-only material: color figures

\section{INTRODUCTION}

In conjunction with the onset of quiescent helium fusion, stars locate on the zero-age horizontal branch (ZAHB) of the H-R diagram. Usually horizontal branch (HB) stars have two energy sources: in addition to the helium burning in their cores, they experience hydrogen fusion in a surrounding shell. The HB domain encompasses a very large effective temperature range, leading to stars being labeled as members of the extreme $\mathrm{HB}$, blue HB, RR Lyrae variables, red HB (RHB), and red clump (RC) stars. The loci of the stars on the HB partly depend on many parameters, including stellar mass, metallicity, age, helium abundance, and rotation, and mass. Mass is a key parameter: HB locations depend sensitively on initial stellar masses or the amount of mass loss encountered during the red giant branch (RGB) phase. Theoretical models (e.g., Sweigart \& Gross 1978; Piersanti et al. 2004) suggest that very low mass stars (around $0.5 M_{\odot}$ ) appear on the blue part of the HB, higher-mass ones (around $0.8 M_{\odot}$ ) sit on the RHB, and stars of solar mass and greater are located in the small $\mathrm{RC}$ area at the extreme red edge of the HB.

RHBs are located between the instability strip and the RC. Stars of the RC concentrate at $M_{\mathrm{V}} \approx 1$ and $B-V \approx 0.9-1$ (Cannon 1970; Wilson 1976). These colors correspond to effective temperatures $T_{\text {eff }} \approx 5000-4800 \mathrm{~K}$ (e.g., Ramírez \& Meléndez 2005), in contrast to the color $/ T_{\text {eff }}$ range of RHB stars $(B-V)_{0} \approx 0.5-0.8, T_{\text {eff }} \approx 5000-6200 \mathrm{~K}$ (Straižys et al. 1981; Gray \& Corbally 2009). It is easy to identify RHB stars in globular cluster (GC) color-magnitude diagrams (e.g., see the survey of Rosenberg et al. 2000a, 2000b) but they are not easily distinguished from RC, subgiant branch (SGB), and even main-sequence (MS) stars in the Galactic field. There have been several attempts to isolate field RHB stars. An early systematic survey was that of Straižys et al. (1981), who observed eight RHB candidates with the Vilnius photometric system. They derived the metallicities and determined the luminosity classes using color-color diagrams, but could not prove their RHB membership because of lack of surface gravity information.

Field RHB stars were first investigated as a group by Rose (1985). He studied G5-G7 stars selected from the Upgren (1962, 1963) North Galactic Pole (NGP) survey. By analyzing lowresolution spectroscopic data, Rose showed that one can distinguish between evolved and "post-MS stars" (i.e., subgiants) using the strengths of $\mathrm{Sr}$ II $\lambda 4077$ and $\mathrm{CN} \lambda 3883$ and 4216 bands. He also estimated the scale height of RHBs to be $>500 \mathrm{pc}$ and concluded that they are moderately metal-poor members of the "thick disk" (Gilmore \& Reid 1983) of the Galaxy.

The RHB assignments subsequently were questioned by Norris (1987), who reported the results of photometric observations of 10 Upgren $(1962,1963)$ stars that formed part of the Rose (1985) RHB candidate sample. Norris argued that the colors of these stars (e.g., $B-V \approx 0.88$ ) are indistinguishable from the RC stars in the old disk, mildly metal-deficient open cluster NGC $2243(t \approx 4$ Gyr; Anthony-Twarog et al. 2005; $[\mathrm{Fe} / \mathrm{H}] \approx-0.4 ;$ Jacobson et al. 2011). In fact, the colors of the Rose candidates have almost no overlap with the colors of true RHB stars $(0.6 \lesssim B-V \lesssim 0.8$; e.g., Hesser et al. 1987) of the GC 47 Tuc $(t \approx 13 \mathrm{Gyr}$; Anthony-Twarog et al. 2005; [Fe/H] = $\approx-0.7$; Hesser et al. 1987).

The evolutionary states of alleged RHB field stars were revisited by Tautvaišienè (1996), who carried out Vilnius system photometric observations of the 13 field RHB candidates from Rose (1985). From these data she deduced spectral types, atmospheric parameters, and absolute magnitudes. Her results yielded a complex message: low mean metallicity $(<[\mathrm{Fe} / \mathrm{H}]>\approx$ $-0.6)$ and large mean age $(t \approx 10-12 \mathrm{Gyr})$ consistent with 
possible RHB status, but low mean temperature $\left(\left\langle T_{\text {eff }}\right\rangle \approx\right.$ $4940 \mathrm{~K}$ ) consistent with the RC.

In another study, Tautvaišiene (1997) gathered highdispersion spectra of 10 field RHB stars that had been identified in a number of previous papers. The metallicity range of her sample was very large, $-0.2 \geqslant[\mathrm{Fe} / \mathrm{H}] \geqslant-1.9$, which resulted in mixed Galactic population membership. She concluded that the chemical compositions of these RHB stars were in accord with those of dwarf and red giant stars of similar metallicities, and thus RHB stars can be used as tracers of the chemical evolution of the Galaxy. Tautvaišienè et al. (2001) followed this paper with another chemical composition study of Rose (1985) stars, finding in particular: (1) overabundances of $\alpha$-elements $(\mathrm{O}, \mathrm{Mg}$, $\mathrm{Si}, \mathrm{Ca}, \mathrm{Ti})$; (2) solar abundances of some neutron-capture elements (Y, Ba, La) and overabundances of others (especially Eu, possibly $\mathrm{Zr}$ and $\mathrm{Sm}$ ); (3) depleted $\mathrm{C}$ and enriched $\mathrm{N}$ abundances, indicative of $\mathrm{CN}$-cycle $\mathrm{H}$-fusion in these stars; and (4) perhaps most importantly, very low carbon isotope ratios, ${ }^{12} \mathrm{C} /{ }^{13} \mathrm{C} \approx$ 3-6.

Previous studies have suggested that true field RHB stars are relatively rare, but the extant sample sizes are small. The ones that exist should be relatively low mass members of the thick disk because stellar evolution models (e.g., Lee et al. 1994) predict that higher-mass, solar-metallicity core Heburning stars should reside exclusively in the RC. Following the Norris (1987) suggestion that "the only way to resolve this problem in an unambiguous way will be to analyze a complete sample of stars having colors in the range $0.7 \leqslant B-V \leqslant$ 0.95," Kaempf et al. (2005) identified a large sample of RHB candidates from the Hipparcos catalog (Perryman et al. 1997). Their kinematic analysis found both thick disk and halo RHBs, and they estimated a Galactic scale height of $0.6 \mathrm{kpc}$ for the thick-disk RHBs.

In this paper we report on a new large-sample chemical composition study of candidate field RHB stars. The combined kinematics, metallicities, and chemical abundance ratios will be used to confirm the existence of a substantial number of high-metallicity thin-disk RHB stars. In Section 2 we present the target star selection and the spectroscopic data that were gathered. Model atmosphere parameter derivation is described in Section 3. We discuss the chemical composition analysis in Section 4, along with comparison to other large-sample literature studies. The kinematics of our sample are given in Section 5, and estimates of their evolutionary states in Section 6. We discuss the implications of these results in Section 7.

\section{OBSERVATIONS}

We obtained high-resolution, high signal-to-noise spectra of stars selected mainly by their colors (as suggested above) and some indications that they might be giants (spectral luminosity class III and/or low $M_{\mathrm{V}}$ from Hipparcos parallax).

\subsection{Selection of the Program Stars}

We assembled our program star list from several sources. Most of our RHB candidates were selected from among the Upgren (1962) NGP survey and the Harlan (1969, 1974, 1981) stars used by Rose (1985). We also made use of SIMBAD 4 and Vizier. $^{5}$

We restricted the prospective sample in color, spectral type, and apparent magnitude $(V<11)$. For spectral type, we limited

\footnotetext{
4 http://simbad.u-strasbg.fr/simbad/

5 http://vizier.u-strasbg.fr/viz-bin/VizieR
}

our search to between G0III and G8III. The reason for this restriction is simple: the loci of the RHB stars in the H-R diagram fall in between the instability strip on the blue side and $\mathrm{RC}$ region on the red side. RHB stars have very similar luminosities to RC stars, although the effective temperatures of RCs are lower. For RC stars, Kaempf et al. (2005) adopted a luminosity-dependent color range of $0.85 \lesssim B-V \lesssim 1.2$ (spectral classes approximately G6-K3), and for RHB stars $0.5 \lesssim B-V \lesssim 0.8$ (approximately G0-G5); see their Figure 1. In our color selection we used this RHB $B-V$ range, but when possible we preferred to use an equivalent $V-K$ range of $\approx 1.5-2.2$, because $V-K$ colors are almost independent of metallicity and gravity but very sensitive to effective temperature. In order to calculate photometric temperatures we have used the metallicity-dependent $T_{\text {eff }}-$ color formula for giants given by Ramírez \& Meléndez (2005). The apparent magnitude limit was defined by our telescope/instrument combination; see Section 2.2. The radial velocity (RV) and proper motion properties of the candidates were not included in the selection criteria, which allowed us to minimize potential kinematic biases in our sample. There is an inevitable bias due to the cutoff in apparent magnitude limit of the instrumental setup.

\subsection{Observations and Data Reduction}

We obtained high-resolution spectra of RHB candidates with the Robert G. Tull Cross-Dispersed Echelle spectrograph (Tull et al. 1995) of the $2.7 \mathrm{~m}$ Harlan J. Smith Telescope at McDonald Observatory. The instrumental setup, including a $1^{\prime \prime} .2$ width entrance slit, yielded a spectral resolving power of $R \equiv \lambda / \Delta \lambda \approx$ 60,000 . Within the wavelength coverage of $\lambda \lambda 3400-10900$, we observed 63 spectral orders. The gaps between the orders reduce toward blue orders, enabling continuous spectral coverage for $\lambda \lesssim 5900 \AA$. The data were obtained during five observing runs corresponding to 22 nights in total.

Based on the selection criteria discussed in Section 2.1, we were able to observe 129 candidate RHB stars with adequate signal to noise. Not all of the stars survived scrutiny after data reduction. Some of these stars were of interest because they were being observed with high spectral resolution for the first time. Inspection of the data revealed that 20 candidates either were rapidly rotating $(V \sin i \geqslant 30)$ or were doublelined spectroscopic binaries. These were discarded from the final sample because in both cases the spectral features become too blended and do not allow us to extract meaningful abundances for this project. In the end we also had to eliminate 33 more candidates during the model atmosphere analyses because we were not be able to obtain reasonable atmospheric parameters from spectroscopic criteria alone (see Section 3 for further discussion). The remaining sample of 76 candidates were studied in detail. In Table 1 we present basic data for these program stars.

The data reductions were performed with the $\mathrm{IRAF}^{6}$ software package in the classical manner: bias subtraction, flat-field division, and scattered light removal followed by extraction of the spectral orders. We took at least two exposures per star and combined them in order to filter out cosmic-ray events. Fastrotating hot stars were observed each night at appropriate air masses, and used to remove telluric features from the spectra of the program stars. Removal of the telluric lines was done with

\footnotetext{
6 The Image Reduction and Analysis Facility, a general purpose software package for astronomical data, is written and supported by the IRAF programming group of the National Optical Astronomy Observatory (NOAO) in Tucson, AZ, USA.
} 


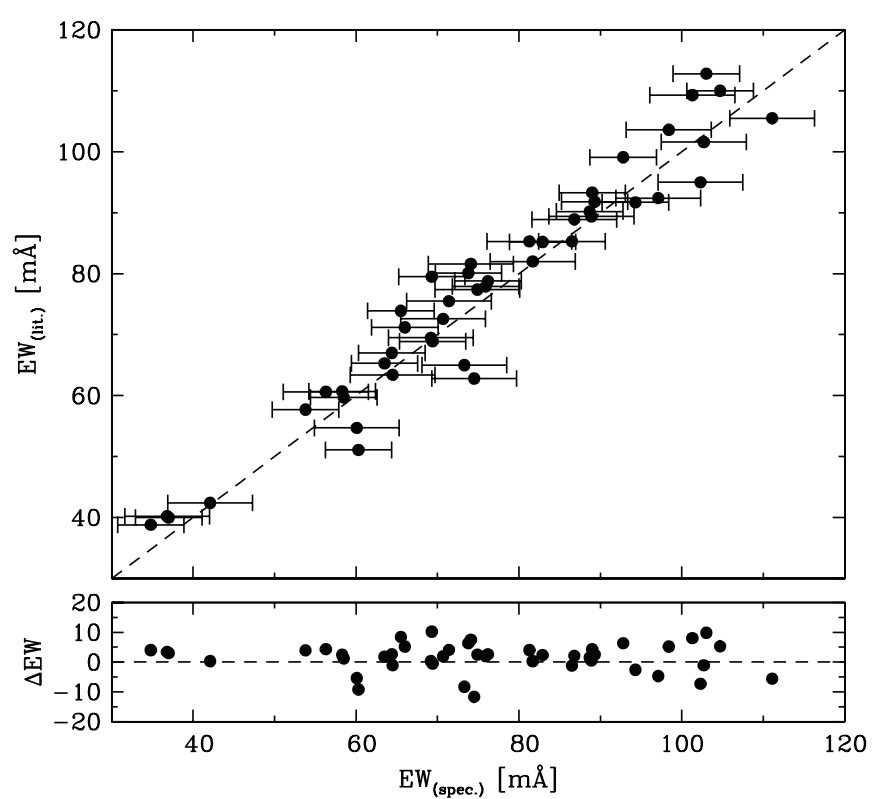

Figure 1. Top panel: comparison of our EWs with previous studies for HIP 13339 and HIP 71837 (Takeda et al. 2005). Bottom panel: differences in EWs, defined as $\Delta \mathrm{EW} \equiv \mathrm{EW}_{\text {literature }}-\mathrm{EW}_{\text {this study. }}$. Error bars represent the $\sigma \simeq 5 \mathrm{~m} \AA$ of $\Delta \mathrm{EW}$.

the IRAF routine telluric, following continuum normalization. ThAr lamp exposures taken at the beginning and end of each night were used for the wavelength calibration, again done with standard IRAF tasks.

Since some of the RHB candidates have reported parallaxes but no RVs, we also observed a few IAU RV standards. We used these standards to measure the RVs of our stars via the cross-correlating technique provided in the fxcor (Fitzpatrick 1993) task in IRAF. The mean RVs of the relevant stars are listed in Table 1. Typical errors for the RV measurements were around $\pm 0.3-0.6 \mathrm{~km} \mathrm{~s}^{-1}$. We also checked our measurements by re-measuring the RVs of a few of our program stars that have previously published values. Our RVs agree with the literature values to within the joint error estimates.

We estimated signal-to-noise ratios $(\mathrm{S} / \mathrm{N})$ of the spectra at three different "continuum" wavelength regions near 4500 , 5500 , and $6500 \AA$ in which we detect no obvious absorption features. We give examples of the derived $\mathrm{S} / \mathrm{N}$ for three program stars with different visual magnitudes in Table 2.

\section{MODEL ATMOSPHERE PARAMETERS}

We have used both equivalent widths (EWs) and spectrum synthesis for the abundance analysis. The line list for model atmospheric parameter determination was generated by choosing clean lines with laboratory $g f$-values between $\lambda 5500$ and 6500 . This spectral region contains many relatively unblended Fe-peak species transitions and only very weak $\mathrm{CN}$ molecular contamination in our program stars. In selecting the transitions we consulted the solar line compendium of Moore et al. (1966) and the Griffin (1968) Arcturus spectral atlas. We measured EWs using the SPECTRE ${ }^{7}$ code (Fitzpatrick \& Sneden 1987). SPECTRE employs a semi-automated routine that fits Voigt and Gaussian line profiles to the observed spectral lines.

In Figure 1 we compare our EW measurements with the values given by Takeda et al. (2005) for HIP 13339 and HIP 71837.

\footnotetext{
7 An interactive spectrum measurement package, available at http://www.as.utexas.edu/ chris/spectre.html.
}

The EW scales are in reasonable agreement: defining $\Delta \mathrm{EW} \equiv$ $\mathrm{EW}_{\text {literature }}-\mathrm{EW}_{\text {this study }}$, we found $\langle\Delta \mathrm{EW}\rangle=+1.6 \mathrm{~m} \AA$, with $\sigma \simeq 5 \mathrm{~mA}$.

Stellar atmosphere models required by the analysis were from the Castelli et al. (1997), Castelli \& Kurucz (2003) $\operatorname{grid}^{8}$ of model atmospheres computed with opacity distribution functions and without convective overshooting. We interpolated in this grid with software developed by Andy McWilliam and Inese Ivans. Then we used an automated version of the spectral line analysis and synthetic spectrum code MOOG (Sneden 1973) $)^{9}$ to determine the abundances of our program stars. MOOG performs analyses using one-dimensional local thermodynamic equilibrium (LTE) equations for plane parallel atmospheres. In the automated version (described in more detail by Hollek et al. 2011; Roederer et al. 2011) all of the steps in model atmosphere derivation and many parts in the relative abundance analysis are done iteratively by the code without human intervention.

We used Fe I and Fe II abundances derived with trial model stellar atmospheres to determine the fundamental stellar parameters of effective temperature $T_{\text {eff }}$, surface gravity, $\log g$, microturbulent velocity $\xi_{\mathrm{t}}$, and metallicity $[\mathrm{Fe} / \mathrm{H}] .{ }^{10}$ Final values of $T_{\text {eff }}$ were estimated by requiring that $\mathrm{Fe}$ I abundances show no trend with excitation potential $\chi$ beyond internal line-to-line scatter uncertainties. A similar approach yielded estimates of $\xi_{\mathrm{t}}$, which is related to small-scale turbulent motion: this parameter was varied until we obtained no apparent Fe I abundance trend with reduced width $\mathrm{RW}$ ( $\equiv \mathrm{EW} / \lambda$ ).

As reported in previous studies, non-local thermodynamical equilibrium (NLTE) mechanism has almost no effect on Fe II lines and has a small effect on Fe I lines for solar-type metallicities and mildly metal-poor stars. As given by Mashonkina et al. (2011) for a temperature range of 4600-6500 K, the departure from LTE is not more than 0.1 dex in Fe I abundances. This value is well within the uncertainty limits of our Fe I abundances (see Section 3.1). Since we have mostly solar-metallicity and mildly metal-poor stars in our program, the corrections for NLTE effects were not applied.

Surface gravities were calculated by demanding that Fe I and Fe II lines yield the same mean abundances to within the $1 \sigma$ internal scatter uncertainties of both species. Finally, we iterated on the $[\mathrm{Fe} / \mathrm{H}]$ metallicities until the values assumed in model creation were consistent with those implied by the line abundance averages. Derived model parameters of our stars are given in Table 3.

We also derived solar abundances (Table 4) applying the same procedure to all the species investigated here. The highresolution solar data were obtained from the electronic version of the solar center-of-disk spectrum of Delbouille et al. (1973). ${ }^{11}$ These abundances were used for differential determination of the stellar abundances. These differential abundances should give more accurate "internal" results than absolute abundances since many of the systematic errors can be nearly compensated in comparing our stars with the Sun. Our internal solar abundances generally agree with those recommended by Asplund et al. (2009), to within the mutual uncertainties. Nitrogen is the sole exception with a substantial difference, $\sim+0.15$ dex, which potentially could lead to a small offset in our $[\mathrm{N} / \mathrm{Fe}]$

\footnotetext{
8 Available at http://kurucz.harvard.edu/grids.html.

9 Available at http://www.as.utexas.edu/ chris/moog.html.

10 For elements $A$ and $B,[A / B]=\log \left(N_{A} / N_{B}\right)_{\star}-\log \left(N_{A} / N_{B}\right)_{\odot}$ and $\log$ $\epsilon(A)=\log \left(N_{A} / N_{H}\right)+12.0$.

11 Available at the Bass2000 Web site, http://bass2000.obspm.fr/.
} 
Table 1

Program Stars

\begin{tabular}{|c|c|c|c|c|c|c|c|c|c|c|}
\hline Stars & $B$ & $V$ & $K_{s}$ & $B-V$ & $V-K_{s}$ & $\begin{array}{c}\pi \\
\text { (mas) }\end{array}$ & $\begin{array}{c}\sigma_{\pi} \\
\text { (mas) }\end{array}$ & 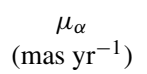 & $\begin{array}{c}\mu_{\delta} \\
\left(\mathrm{mas} \mathrm{yr}^{-1}\right)\end{array}$ & $\mathrm{RV}$ \\
\hline HIP 476 & 6.43 & 5.55 & 3.77 & 0.88 & 1.78 & 8.75 & 0.30 & 43.58 & -5.96 & $\ldots$ \\
\hline HIP 3031 & 5.24 & 4.40 & 2.07 & 0.84 & 2.33 & 19.91 & 0.19 & -229.04 & -253.11 & $\ldots$ \\
\hline HIP 4197 & 7.56 & 6.71 & 4.51 & 0.85 & 2.20 & 4.78 & 1.16 & -2.54 & -46.24 & $\ldots$ \\
\hline HIP 4960 & 9.31 & 8.60 & 6.58 & 0.71 & 2.03 & 3.18 & 0.93 & 13.62 & -20.59 & $\ldots$ \\
\hline HIP 5104 & 8.40 & 7.65 & 5.59 & 0.75 & 2.06 & 3.19 & 0.79 & 63.61 & 49.81 & $\ldots$ \\
\hline HIP 8404 & 6.88 & 5.91 & 3.86 & 0.97 & 2.05 & 10.16 & 0.47 & -7.40 & 19.26 & $\ldots$ \\
\hline HIP 11924 & 8.63 & 8.00 & 6.50 & 0.63 & 1.50 & 11.02 & 0.77 & -62.76 & -57.96 & $\ldots$ \\
\hline HIP 13339 & 6.75 & 5.87 & 3.85 & 0.88 & 2.02 & 8.34 & 0.37 & -25.49 & -24.74 & $\ldots$ \\
\hline HIP 19611 & 7.60 & 6.60 & 4.44 & 1.00 & 2.17 & 6.87 & 0.77 & -11.57 & 14.79 & 4.0 \\
\hline HIP 19740 & 5.71 & 4.89 & 2.97 & 0.82 & 1.92 & 9.83 & 0.64 & -10.31 & -30.01 & $\ldots$ \\
\hline HIP 27280 & 6.67 & 5.79 & 3.93 & 0.88 & 1.86 & 10.95 & 0.50 & -32.08 & -65.50 & $\ldots$ \\
\hline HIP 38801 & 9.14 & 8.48 & 6.83 & 0.66 & 1.65 & 1.64 & 1.35 & -2.02 & 1.08 & 11.1 \\
\hline HIP 39326 & 6.91 & 6.30 & 4.82 & 0.61 & 1.48 & 8.71 & 0.40 & 6.87 & -10.52 & $\ldots$ \\
\hline HIP 44154 & 6.13 & 5.23 & 3.15 & 0.90 & 2.08 & 11.03 & 0.28 & -43.78 & -35.03 & $\ldots$ \\
\hline HIP 45033 & 6.90 & 6.03 & 4.09 & 0.87 & 1.94 & 8.86 & 0.42 & 2.64 & 5.37 & $\ldots$ \\
\hline HIP 45158 & 6.68 & 5.73 & 3.54 & 0.95 & 2.19 & 10.73 & 0.37 & -56.68 & 45.79 & $\ldots$ \\
\hline HIP 45412 & 6.80 & 5.99 & 3.86 & 0.81 & 2.13 & 7.69 & 0.54 & -158.20 & 47.73 & $\ldots$ \\
\hline HIP 46325 & 9.13 & 8.38 & 6.76 & 0.74 & 1.63 & 11.28 & 1.00 & 13.88 & -150.81 & $\ldots$ \\
\hline HIP 51179 & 7.49 & 6.65 & 4.53 & 0.84 & 2.12 & 8.05 & 0.56 & -38.90 & 14.13 & -25.2 \\
\hline HIP 51487 & 10.33 & 9.53 & 7.64 & 0.80 & 1.89 & 16.89 & 1.23 & -6.09 & -33.43 & -14.5 \\
\hline HIP 54048 & 7.21 & 6.35 & 4.32 & 0.86 & 2.03 & 6.04 & 0.46 & 5.18 & -5.06 & -6.3 \\
\hline HIP 56194 & 7.47 & 6.55 & 4.41 & 0.93 & 2.14 & 5.66 & 0.41 & -25.08 & -25.49 & $\ldots$ \\
\hline HIP 57535 & 9.28 & 8.60 & 7.06 & 0.68 & 1.54 & 5.51 & 0.86 & 39.71 & -7.07 & $\ldots$ \\
\hline HIP 57748 & 8.66 & 7.91 & 6.01 & 0.75 & 1.90 & 4.47 & 0.74 & 23.31 & -19.18 & -22.0 \\
\hline HIP 58269 & 9.41 & 8.70 & 7.18 & 0.71 & 1.51 & 10.32 & 1.12 & 80.71 & -144.32 & $\ldots$ \\
\hline HIP 60140 & 8.50 & 7.67 & 5.60 & 0.83 & 2.07 & 3.75 & 0.79 & -15.08 & 3.63 & 0.9 \\
\hline HIP 60485 & 5.64 & 4.77 & 2.82 & 0.87 & 1.96 & 8.44 & 0.24 & 12.64 & 11.43 & $\ldots$ \\
\hline HIP 60873 & 9.61 & 8.81 & 6.74 & 0.80 & 2.07 & 5.39 & 1.09 & -58.88 & 2.83 & -32.7 \\
\hline HIP 62325 & 6.66 & 5.70 & 3.44 & 0.96 & 2.26 & 22.15 & 0.35 & 279.34 & -453.41 & $\ldots$ \\
\hline HIP 65900 & 8.93 & 8.34 & 7.00 & 0.59 & 1.34 & 16.74 & 0.91 & -132.28 & -29.61 & $\ldots$ \\
\hline HIP 66892 & 7.16 & 6.30 & 4.26 & 0.86 & 2.04 & 3.58 & 0.40 & -85.88 & 13.24 & $\ldots$ \\
\hline HIP 70341 & 9.45 & 8.82 & 7.20 & 0.63 & 1.62 & 14.36 & 1.02 & -147.37 & -24.82 & $\ldots$ \\
\hline HIP 70344 & 7.80 & 7.22 & 5.79 & 0.58 & 1.43 & 14.20 & 0.78 & 20.49 & -151.26 & $\ldots$ \\
\hline HIP 71837 & 6.50 & 5.56 & 3.41 & 0.94 & 2.15 & 8.09 & 0.33 & -158.78 & -112.62 & $\ldots$ \\
\hline HIP 72631 & 5.91 & 4.90 & 2.80 & 1.01 & 2.10 & 14.92 & 0.40 & 89.97 & -124.57 & $\ldots$ \\
\hline HIP 75823 & 9.33 & 8.54 & 6.44 & 0.79 & 2.10 & 2.49 & 1.16 & -23.31 & 9.96 & $\ldots$ \\
\hline HIP 78990 & 5.14 & 4.33 & 2.47 & 0.81 & 1.86 & 11.22 & 0.32 & 44.81 & -45.42 & $\ldots$ \\
\hline HIP 80309 & 6.63 & 5.67 & 3.68 & 0.96 & 1.99 & 6.44 & 0.32 & -29.31 & 34.26 & $\ldots$ \\
\hline HIP 80543 & 7.30 & 6.69 & 5.22 & 0.61 & 1.47 & 12.56 & 0.55 & -0.46 & -3.42 & $\ldots$ \\
\hline HIP 82014 & 9.67 & 8.92 & 7.14 & 0.75 & 1.78 & 8.23 & 0.80 & -48.14 & 119.81 & -19.9 \\
\hline HIP 85715 & 6.58 & 5.64 & 3.57 & 0.95 & 2.07 & 8.27 & 0.27 & 3.77 & 16.33 & $\ldots$ \\
\hline HIP 89008 & 6.49 & 5.57 & 3.65 & 0.92 & 1.92 & 8.28 & 0.30 & 12.72 & 11.12 & $\ldots$ \\
\hline HIP 89095 & 7.98 & 7.13 & 5.12 & 0.85 & 2.01 & 2.73 & 0.51 & 8.25 & 14.35 & $\ldots$ \\
\hline HIP 90906 & 8.33 & 7.44 & 5.29 & 0.89 & 2.15 & 3.39 & 0.73 & 4.63 & 14.69 & $\ldots$ \\
\hline HIP 91985 & 7.07 & 6.26 & 4.38 & 0.81 & 1.88 & 3.35 & 0.30 & 9.93 & -2.23 & $\ldots$ \\
\hline HIP 92827 & 8.46 & 7.63 & 5.45 & 0.83 & 2.18 & 5.06 & 0.60 & 20.70 & 21.85 & $\ldots$ \\
\hline HIP 93940 & 8.79 & 8.25 & 6.88 & 0.54 & 1.37 & 7.81 & 0.83 & 37.43 & 7.16 & -53.4 \\
\hline HIP 94598 & 6.91 & 6.04 & 4.05 & 0.87 & 1.99 & 4.79 & 0.42 & 3.33 & -10.99 & $\ldots$ \\
\hline HIP 94779 & 4.73 & 3.80 & 1.76 & 0.93 & 2.04 & 26.27 & 0.10 & 60.07 & 122.83 & $\ldots$ \\
\hline HIP 98587 & 8.55 & 7.70 & 5.65 & 0.85 & 2.05 & 2.68 & 0.67 & 3.52 & -26.95 & $\ldots$ \\
\hline HIP 100274 & 7.07 & 6.17 & 4.25 & 0.90 & 1.93 & 5.88 & 0.52 & -3.10 & 6.71 & $\ldots$ \\
\hline HIP 103004 & 5.39 & 4.58 & 2.72 & 0.81 & 1.86 & 17.30 & 0.66 & -75.44 & -62.03 & $\ldots$ \\
\hline HIP 103734 & 6.99 & 6.01 & 4.04 & 0.98 & 1.97 & 5.21 & 0.33 & -3.47 & -6.42 & $\ldots$ \\
\hline HIP 113610 & 7.10 & 6.23 & 4.22 & 0.87 & 2.00 & 5.57 & 0.69 & 44.21 & 9.99 & -14.7 \\
\hline HIP 114809 & 7.64 & 6.80 & 4.76 & 0.84 & 2.04 & 6.22 & 0.45 & -0.29 & 12.71 & $\ldots$ \\
\hline HIP 115839 & 7.26 & 6.38 & 4.33 & 0.88 & 2.05 & 7.97 & 0.42 & 99.16 & -21.66 & $\ldots$ \\
\hline HIP 118209 & 5.81 & 4.89 & 2.95 & 0.92 & 1.94 & 13.91 & 0.28 & -57.13 & -72.08 & $\ldots$ \\
\hline HD 9097 & 10.76 & 10.20 & 8.51 & 0.56 & 1.69 & $\ldots$ & $\ldots$ & $\ldots$ & $\ldots$ & $\ldots$ \\
\hline HD 84686 & 9.40 & 8.55 & 6.44 & 0.85 & 2.11 & $\ldots$ & $\ldots$ & $\ldots$ & $\cdots$ & $\ldots$ \\
\hline HD 96780 & 9.31 & 8.80 & 7.52 & 0.51 & 1.28 & $\cdots$ & $\ldots$ & $\ldots$ & $\cdots$ & $\ldots$ \\
\hline HD 101014 & 10.00 & 9.40 & 8.02 & 0.60 & 1.38 & $\ldots$ & $\ldots$ & $\ldots$ & $\ldots$ & $\ldots$ \\
\hline HD 141770 & 9.53 & 8.88 & 7.09 & 0.65 & 1.80 & $\ldots$ & $\ldots$ & $\ldots$ & $\ldots$ & $\ldots$ \\
\hline HD 166310 & 10.25 & 9.54 & 7.66 & 0.71 & 1.88 & $\ldots$ & $\ldots$ & $\ldots$ & $\ldots$ & $\ldots$ \\
\hline HD 221744 & 9.84 & 9.22 & 7.50 & 0.62 & 1.72 & $\ldots$ & $\ldots$ & $\ldots$ & $\ldots$ & $\ldots$ \\
\hline HD 235802 & 9.78 & 9.06 & 7.28 & 0.72 & 1.78 & $\ldots$ & $\ldots$ & $\ldots$ & $\ldots$ & $\ldots$ \\
\hline
\end{tabular}


Table 1

(Continued)

\begin{tabular}{lrrrrrrrrr}
\hline \hline Stars & $B$ & $V$ & $K_{s}$ & $B-V$ & $V-K_{s}$ & $\begin{array}{c}\pi \\
(\mathrm{mas})\end{array}$ & $\begin{array}{c}\sigma_{\pi} \\
(\mathrm{mas})\end{array}$ & $\begin{array}{c}\mu_{\alpha} \\
\left(\mathrm{mas} \mathrm{yr}^{-1}\right)\end{array}$ \\
\hline HD 242647 & 10.31 & 9.70 & 8.23 & 0.61 & 1.47 & $\ldots$ & $\ldots$ & $\ldots$ & $\ldots$ \\
$\left(\mathrm{mas} \mathrm{yr}^{-1}\right)$
\end{tabular}

Table 2

$\mathrm{S} / \mathrm{N}$ Ratios

\begin{tabular}{lcccc}
\hline \hline Stars & $V$ & \multicolumn{3}{c}{$\mathrm{S} / \mathrm{N}$} \\
\cline { 3 - 5 } & & $4500 \AA$ & $5500 \AA$ & $6500 \AA$ \\
\hline BD+27 2057 & 10.43 & 120 & 170 & 160 \\
HD 84686 & 9.4 & 95 & 200 & 190 \\
HIP 98587 & 8.55 & 115 & 250 & 210 \\
\hline
\end{tabular}

results compared to the previous studies that we will consider in Section 4.3. Our adopted CN oscillator strengths from the Kurucz database may be the major factor contributing to this offset. Exploration of this issue in detail is beyond the scope of our paper.

\subsection{Uncertainties in Parameters}

The internal uncertainties in atmospheric parameters $T_{\text {eff }}$, $\log g$, and $\xi_{\mathrm{t}}$ were estimated by running a series of trial analyses on the spectral data of BD+27 2057, HD 84686, and HIP 98587. For temperature uncertainties, we varied the assumed $T_{\text {eff }}$ in steps of $50 \mathrm{~K}$ and kept the other parameters fixed during the analysis. The temperature was changed until the mean abundance difference between low-and high-excitation Fe I lines exceeded the $\pm 1 \sigma$ scatter of individual line abundances derived with the optimal $T_{\text {eff }}$, in other words, until Fe I abundances showed an obviously unacceptable trend with excitation potential. This method yielded an average uncertainty of $\sim 150 \mathrm{~K}$ for the $T_{\text {eff }}$.

To estimate the external uncertainty in $T_{\text {eff }}$ we have searched the literature for previous high-resolution studies of our program stars. We found no large samples in common with our stars, but from the $T_{\text {eff }}$ values reported in various publications we have generated Figure 2. The heterogeneity of the literature data does not justify a detailed statistical treatment, but it is clear that our $T_{\text {eff }}$ values track those in previous publications, with a scatter of $\sim 120 \mathrm{~K}$. We adopt an overall $T_{\text {eff }}$ uncertainty of $\pm 150 \mathrm{~K}$.

Our $T_{\text {eff }}$ values for a few stars, however, deviate significantly from previous estimates. Since temperature estimates are critical to the assessment of RHB or RC status for our stars, we turned to the spectroscopic "Line Depth Ratio" (LDR) method of $T_{\text {eff }}$ determinations. Gray \& Brown (2001) showed simple LDRs of several line pairs in the $6200 \AA$ spectral region (most often a ratio formed by comparing the central depths of a $\mathrm{V}_{\mathrm{I}}$ and an Fe I line). Their paper maps the LDRs to stellar $B-V$ values and those colors in turn to $T_{\text {eff }}$ estimates. LDRs are excellent temperature indicators especially for giants with spectral types between G3 and K3 (see also Figure 7 of Gray \& Brown

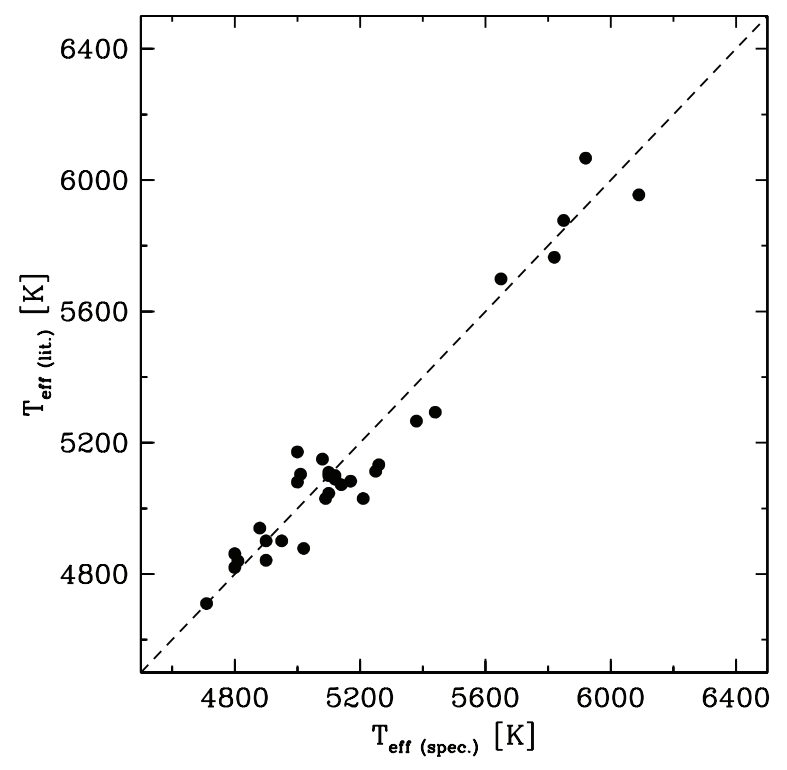

Figure 2. Comparison of our derived spectroscopic $T_{\text {eff }}$ values with those gathered from heterogeneous literature (filled circles): Cottrell \& Sneden (1986), McWilliam (1990), Ryan \& Lambert (1995), Mallik (1998), Chen et al. (2000), Tautvaišiené et al. (2001), Franchini et al. (2004), Nordström et al. (2004), Luck $\&$ Heiter (2007), Robinson et al. (2007), Soubiran et al. (2008), Takeda et al. (2008), Omiya et al. (2009), and Liu et al. (2010).

2001). This method produces effective temperatures well within the uncertainty levels of $150 \mathrm{~K}$ and gives us an independent spectroscopic check on our spectroscopic $T_{\text {eff }}$ estimates from Fe I lines.

We measured LDRs for our whole sample. We mainly made use of the line ratio pairs recommended by Gray \& Brown $(2001)^{12}$ and derived an average $T_{\text {eff }}(\mathrm{LDR})$ for the stars. These line pairs compare the central depths of a very $T_{\text {eff }}$-sensitive transition (almost always a low-excitation VI line) usually with a high-excitation transition of another Fe-group species. We especially made use of the $6224.5 \AA / 6223.9 \AA$ pair that compares a V I line with $\chi=0.29 \mathrm{eV}$ to an $\mathrm{Ni}$ I line with $\chi=4.10 \mathrm{eV}$. Their LDR was the most useful because it could be reliably measured throughout most of the $T_{\text {eff }}$ domain of our sample. However, the depths of the V I lines weaken rapidly with increasing temperature $(\geqslant 5500 \mathrm{~K})$. For example,

\footnotetext{
12 The line ratio pairs used for LDR temperatures: $\mathrm{V}_{\mathrm{I}}(6224.5 \AA) / \mathrm{Ni} \mathrm{I}(6223.9 \AA), \mathrm{V} \mathrm{I}(6233.2 \AA) / \mathrm{Fe} \mathrm{I}(6232.6 \AA)$,

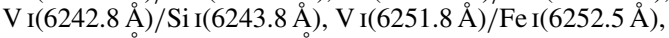
$\mathrm{V}_{\mathrm{I}}(6256.8 \AA) / \mathrm{Fe} \mathrm{I}(6255.9 \AA)$.
} 
Table 3

Model Atmosphere Parameters

\begin{tabular}{|c|c|c|c|c|}
\hline Star & $\begin{array}{l}T_{\text {eff }} \\
(\mathrm{K})\end{array}$ & $\log g$ & $\begin{array}{c}v_{t} \\
\left(\mathrm{~km} \mathrm{~s}^{-1}\right)\end{array}$ & {$[\mathrm{Fe} / \mathrm{H}]$} \\
\hline HIP 476 & 5140 & 2.80 & 1.35 & -0.03 \\
\hline HIP 3031 & 5020 & 2.60 & 1.45 & -0.53 \\
\hline HIP 4197 & 4800 & 2.50 & 1.00 & -0.23 \\
\hline HIP 4960 & 5260 & 2.55 & 1.60 & -1.02 \\
\hline HIP 5104 & 5100 & 2.50 & 1.55 & -0.86 \\
\hline HIP 8404 & 4800 & 2.35 & 1.20 & -0.05 \\
\hline HIP 11924 & 5850 & 4.00 & 1.00 & 0.30 \\
\hline HIP 13339 & 5120 & 2.85 & 0.95 & 0.23 \\
\hline HIP 19611 & 5080 & 2.90 & 1.25 & 0.00 \\
\hline HIP 19740 & 5100 & 2.40 & 1.20 & -0.03 \\
\hline HIP 27280 & 5100 & 2.95 & 1.10 & 0.05 \\
\hline HIP 38801 & 5740 & 2.70 & 2.10 & -0.06 \\
\hline HIP 39326 & 5750 & 3.15 & 1.60 & -0.21 \\
\hline HIP 44154 & 5000 & 2.60 & 1.20 & 0.04 \\
\hline HIP 45033 & 5120 & 2.75 & 1.10 & 0.02 \\
\hline HIP 45158 & 4900 & 2.70 & 1.40 & -0.23 \\
\hline HIP 45412 & 5080 & 2.55 & 1.55 & -0.68 \\
\hline HIP 46325 & 5650 & 4.15 & 1.00 & 0.46 \\
\hline HIP 51179 & 5020 & 2.80 & 1.10 & -0.15 \\
\hline HIP 51487 & 5440 & 4.55 & 1.30 & -0.17 \\
\hline HIP 54048 & 5100 & 2.65 & 1.20 & 0.00 \\
\hline HIP 56194 & 4970 & 2.70 & 1.20 & 0.15 \\
\hline HIP 57535 & 5700 & 3.70 & 1.30 & 0.10 \\
\hline HIP 57748 & 5320 & 2.50 & 1.75 & -0.08 \\
\hline HIP 58269 & 5820 & 4.30 & 1.10 & 0.35 \\
\hline HIP 60140 & 4930 & 2.65 & 0.85 & -0.08 \\
\hline HIP 60485 & 5210 & 2.65 & 1.65 & 0.08 \\
\hline HIP 60873 & 5080 & 3.25 & 0.90 & -0.47 \\
\hline HIP 62325 & 4710 & 2.85 & 0.90 & -0.06 \\
\hline HIP 65900 & 6090 & 4.40 & 1.00 & 0.15 \\
\hline HIP 66892 & 5170 & 2.70 & 1.35 & -0.02 \\
\hline HIP 70341 & 5700 & 4.40 & 0.90 & 0.00 \\
\hline HIP 70344 & 5950 & 3.70 & 1.25 & -0.04 \\
\hline HIP 71837 & 4900 & 2.60 & 1.10 & -0.15 \\
\hline HIP 72631 & 4800 & 2.60 & 1.20 & -0.21 \\
\hline HIP 75823 & 5200 & 2.85 & 1.10 & -0.29 \\
\hline HIP 78990 & 5380 & 2.65 & 1.55 & 0.10 \\
\hline HIP 80309 & 5000 & 2.75 & 1.50 & -0.06 \\
\hline HIP 80543 & 5920 & 3.65 & 1.40 & 0.15 \\
\hline HIP 82014 & 5490 & 3.90 & 1.00 & 0.13 \\
\hline HIP 85715 & 4950 & 2.50 & 1.50 & 0.00 \\
\hline HIP 89008 & 5000 & 2.55 & 1.15 & 0.14 \\
\hline HIP 89095 & 5270 & 2.40 & 1.55 & 0.14 \\
\hline HIP 90906 & 4930 & 2.35 & 1.20 & 0.00 \\
\hline HIP 91985 & 5290 & 2.30 & 1.60 & -0.06 \\
\hline HIP 92827 & 4850 & 2.70 & 0.85 & 0.04 \\
\hline HIP 93940 & 6110 & 3.80 & 1.30 & 0.20 \\
\hline HIP 94598 & 5010 & 2.45 & 1.30 & -0.05 \\
\hline HIP 94779 & 4880 & 2.60 & 1.15 & 0.19 \\
\hline HIP 98587 & 4930 & 2.60 & 1.00 & -0.16 \\
\hline HIP 100274 & 5010 & 2.65 & 1.15 & 0.08 \\
\hline HIP 103004 & 5250 & 2.80 & 1.35 & -0.09 \\
\hline HIP 103734 & 5060 & 2.65 & 1.25 & 0.31 \\
\hline HIP 113610 & 5140 & 2.55 & 1.45 & 0.00 \\
\hline HIP 114809 & 5120 & 2.65 & 1.20 & -0.26 \\
\hline HIP 115839 & 5000 & 2.80 & 1.30 & -0.40 \\
\hline HIP 118209 & 5090 & 2.90 & 1.25 & 0.15 \\
\hline HD 9097 & 5540 & 4.10 & 0.80 & 0.18 \\
\hline HD 96780 & 6200 & 4.00 & 1.30 & 0.01 \\
\hline HD 84686 & 5180 & 2.60 & 1.75 & -0.05 \\
\hline HD 101014 & 5880 & 4.20 & 0.80 & 0.30 \\
\hline HD 141770 & 5650 & 4.05 & 1.05 & 0.09 \\
\hline HD 166310 & 5480 & 4.60 & 1.35 & 0.17 \\
\hline HD 221744 & 5850 & 4.40 & 1.05 & 0.00 \\
\hline HD 235802 & 5680 & 4.35 & 1.25 & 0.25 \\
\hline
\end{tabular}

Table 3

(Continued)

\begin{tabular}{lcccr}
\hline \hline Star & $\begin{array}{c}T_{\text {eff }} \\
(\mathrm{K})\end{array}$ & $\log g$ & $\begin{array}{c}v_{t} \\
\left(\mathrm{~km} \mathrm{~s}^{-1}\right)\end{array}$ & {$[\mathrm{Fe} / \mathrm{H}]$} \\
\hline HD 242647 & 5890 & 4.30 & 0.95 & -0.20 \\
HD 243170 & 6100 & 3.70 & 1.10 & 0.15 \\
BD-14 1413 & 5900 & 3.20 & 1.60 & -0.10 \\
BD+27 2057 & 4810 & 2.25 & 1.25 & -0.51 \\
BD+31 2565 & 6150 & 4.30 & 1.15 & 0.03 \\
BD+32 2190 & 5860 & 4.25 & 0.80 & 0.29 \\
BD+41 2221 & 5810 & 4.15 & 1.15 & 0.22 \\
BD+45 1958 & 5570 & 4.40 & 0.90 & 0.24 \\
BD+45 2032 & 5420 & 3.70 & 0.80 & -0.16 \\
BD+54 2710 & 4840 & 2.20 & 1.45 & 0.04 \\
TYC 3720-324-1 & 5960 & 4.20 & 1.05 & 0.38 \\
\hline
\end{tabular}

Table 4

The Adopted Solar Abundances

\begin{tabular}{|c|c|c|}
\hline Species & $\begin{array}{c}\log \epsilon(\mathrm{X}) \\
(\operatorname{dex})\end{array}$ & $\begin{array}{c}\sigma \\
(\mathrm{dex})\end{array}$ \\
\hline $\mathrm{Li}$ I & 1.02 & \\
\hline $\mathrm{C}_{\mathrm{I}}$ & 8.53 & 0.06 \\
\hline $\mathrm{C}(\mathrm{CH} 4300)$ & 8.38 & \\
\hline $\mathrm{N}(\mathrm{CN} 8000-8040)$ & 8.2 & \\
\hline O I (7774) & 8.91 & 0.01 \\
\hline [O I $](6300)$ & 8.64 & \\
\hline Si I & 7.55 & 0.14 \\
\hline $\mathrm{CaI}$ & 6.24 & 0.08 \\
\hline $\mathrm{Fe} \mathrm{I}$ & 7.5 & 0.08 \\
\hline Fe II & 7.5 & 0.07 \\
\hline La II & 1.17 & \\
\hline Eu II & 0.52 & \\
\hline
\end{tabular}

for the five V I lines recommended by Gray \& Brown (2001) for LDR studies, $\langle\mathrm{EW}\rangle \simeq 6 \mathrm{~m} \AA$ in the solar spectrum (Moore et al. 1966). Lines with such small EWs are near the reliable detection/measurement limit of our spectra. Therefore for warmer stars of our sample we also measured some LDRs proposed by Strassmeier \& Schordan (2000).

A comparison of our spectroscopic and LDR temperatures is given in Figure 3. The error bar depicted in this figure corresponds to the standard deviation of the mean difference between these two temperatures, $\sigma \simeq \pm 150 \mathrm{~K}$. A change in the correlation is apparent at $T_{\text {eff }} \sim 5500 \mathrm{~K}$, which we have highlighted by using different colors for the symbols of stars warmer and cooler than this $T_{\text {eff }}$. Although we see a small systematic bias toward higher temperatures for $T_{\text {eff }}<5500 \mathrm{~K}$ (Figure 3, purple points), the standard deviation of these points is around $90 \mathrm{~K}$ and well within the uncertainty limits. We suggest that this systematic deviation may both arise from the method we adopt from Gray \& Brown (2001), which first involves a calibration of LDR against $B-V$ color indices, then converts these into temperatures, and LDRs that we obtained with instrumentation different from those of Gray \& Brown (2001). As mentioned above, this temperature is near the reliability limit for the LDR method using these transitions. On the other hand, for $T_{\text {eff }}<5500 \mathrm{~K}$, which proves to be the more important domain for identifying true RHB targets, these two $T_{\text {eff }}$ estimators are considerably in good agreement. This result suggests that for stars when clashes occur between photometric and spectroscopic temperatures, the photometric values are probably not reliable for $T_{\text {eff }}<5500 \mathrm{~K}$. In general, the LDR method supports our claimed temperatures for the stars, 


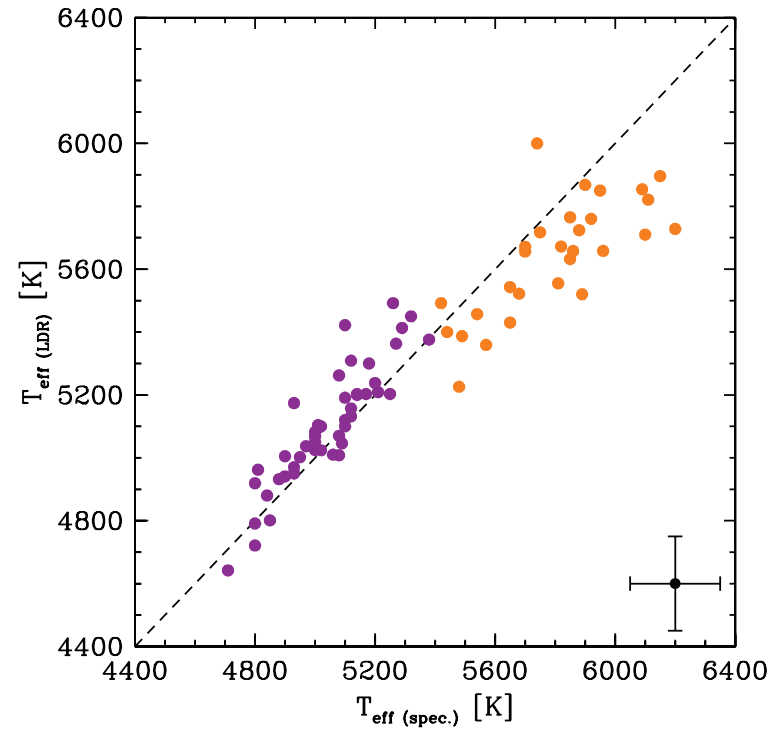

Figure 3. Comparison of our spectroscopic $T_{\text {eff }}$ with the ones obtained using LDR method. A break in the relation is seen at $T_{\text {eff }}<5500 \mathrm{~K}$, which we call attention to by using different symbol colors for stars warmer and cooler than this temperature; see the text for discussion of this issue.

(A color version of this figure is available in the online journal.)

including the ones in which our spectroscopic values clash with literature estimates.

We determined internal uncertainties for $\log g$ and $\xi_{\mathrm{t}}$ through repeated trials with variations in these quantities. The typical average uncertainties were estimated as 0.16 dex and $0.2 \mathrm{~km} \mathrm{~s}^{-1}$, respectively. Comparison of our $\log g$ results with previously reported ones resulted in an external uncertainty level of $\approx 0.25$ dex. By taking into account both internal and external uncertainty levels, we adopt an average uncertainty for $\log g$ of $\approx \pm 0.3$ dex.

We also calculated "physical" gravities and compared them with spectroscopic gravities in order to possibly gain insight on the masses of our stars. We use the following standard equation for physical gravities:

$$
\begin{aligned}
\log g_{\star}= & 0.4\left(M_{\mathrm{V} \star}+B C-M_{\mathrm{Bol} \odot}\right)+\log g_{\odot} \\
& +4 \log \left(\frac{T_{\mathrm{eff} \star}}{T_{\mathrm{eff} \odot}}\right)+\log \left(\frac{m_{\star}}{m_{\odot}}\right) .
\end{aligned}
$$

For the Sun, we adopted $T_{\text {eff }}=5780 \mathrm{~K}, \log g=4.44 \mathrm{dex}$, and $M_{\text {Bol } \odot}=4.75 \mathrm{mag}$. Bolometric corrections were calculated using the relation given by Alonso et al. (1999). A comparison of physical gravities, $\log g$ (theo.), with spectroscopic gravities, $\log g$ (spec.), is given in Figure 4. A significant uncertainty in a physical gravity calculation is the assumed mass. Therefore in the figure we give three $\log g$ (theo.) values calculated for the masses of 1,2 , and $4 M_{\odot}$. They are shown with dashed and dotted lines. The best average agreement between these two $\log g$ scales for significantly evolved stars $(\log g \lesssim 3.0)$ is for $M \simeq 2 M_{\odot}$ and for $\mathrm{MS} / \mathrm{SG}$ stars it is $M \simeq 2.5 M_{\odot}$. The joint uncertainties are too large to draw conclusions about individual stars, but does suggest that our sample is not dominated by high-mass stars.

In order to derive the effect of these uncertainties on abundance determinations, we did multiple analyses by changing $T_{\text {eff }}, \log g,[\mathrm{Fe} / \mathrm{H}]$, and $\xi_{\mathrm{t}}$ within their uncertainty limits. The effective temperature uncertainties create an uncertainty around 0.15-0.16 dex in Fe I and the uncertainties in $\log g$ make the

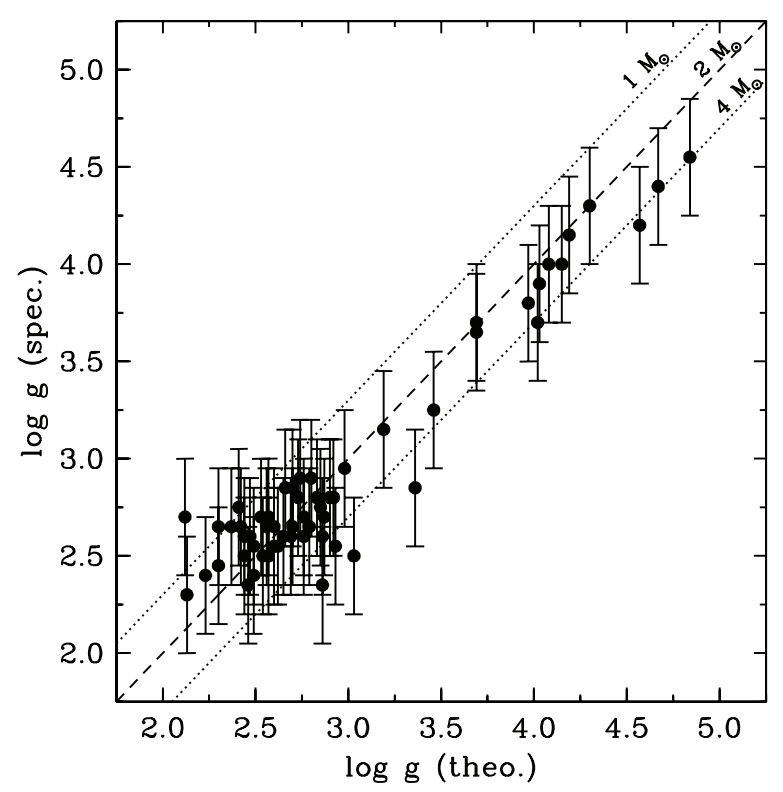

Figure 4. Comparison of our spectroscopic $\log g$ values with theoretical ones. $\log g$ (theo.) are calculated for $M=1,2$, and $4 M_{\odot}$ (dashed and dotted lines). The uncertainty for our spectral $\log g$ is \pm 0.3 dex. We do not have errors on the theoretical values since those are adopted masses.

Fe II abundances change in less than 0.1 dex. The other elements' abundances vary between 0.1 and 0.16 dex due to $T_{\text {eff }}$ uncertainties. In general, the uncertainties in surface gravity and microturbulent velocity result in abundance uncertainties less than $\simeq 0.1$ dex.

Finally, we comment on the uncertainties in our derived ${ }^{12} \mathrm{C} /{ }^{13} \mathrm{C}$ ratios. These are nearly insensitive to model atmosphere uncertainties, since ${ }^{12} \mathrm{CN}$ and ${ }^{13} \mathrm{CN}$ are nearly identical molecules. We estimated the ${ }^{12} \mathrm{C} /{ }^{13} \mathrm{C}$ uncertainties by fitting synthetic spectra to the observed ${ }^{12} \mathrm{CN}$ and ${ }^{13} \mathrm{CN}$ features with various isotopic ratios to estimate their maximum and minimum probable values. The error estimates for the ${ }^{12} \mathrm{C} /{ }^{13} \mathrm{C}$ ratios are given in Table 5.

\subsection{Photometric Temperatures and Reddening}

Since we observed almost half of our program stars at high spectral resolution for the first time, our sample lacked a consistent set of reported atmospheric parameters. This made it difficult to have meaningful temperature estimates to start our analyses, so we relied exclusively on our Fe I line analyses to derive $T_{\text {eff }}$ values, augmenting these with LDR estimates. Armed with these results, we now compare spectroscopic and photometric temperatures for the stars in common. We concentrate on the $V-K$ color because it is one of the best photometric temperature indicators, having almost no dependence on metallicity and gravity (e.g., Alonso et al. 1999; Ramírez \& Meléndez 2005). The $V$ magnitudes that we employ are on the standard Johnson photometric system; the $K_{s}$ magnitudes are from the Two Micron All Sky Survey. We adopt the $\left(V-K_{s}\right)-T_{\text {eff }}$ calibration equation from Ramírez \& Meléndez (2005).

Before calculating the photometric $T_{\text {eff }}$ values, we first applied interstellar reddening corrections to the $V-K_{s}$ colors. A recent survey of the regions within $300 \mathrm{pc}$ of the solar system showed that the "Local Bubble" (Lallement et al. 2003) has a shape of an irregular lacuna, which extends $\sim 60$ pc toward the Galactic center, $80-150$ pc toward the outermost edge of the Galaxy, and $\sim 200$ pc above and below the Galactic plane. 
Table 5

${ }^{12} \mathrm{C} /{ }^{13} \mathrm{C}$ Ratios and Their Uncertainties

\begin{tabular}{|c|c|c|}
\hline Stars & ${ }^{12} \mathrm{C} /{ }^{13} \mathrm{C}$ & $\sigma$ \\
\hline HIP 45412 & 5 & $+2 /-1.5$ \\
\hline HIP 115839 & 5 & $+2 /-1$ \\
\hline BD+27 2057 & 5 & $+2 /-1$ \\
\hline HIP 57748 & 5 & $+2 /-1$ \\
\hline HIP 4960 & $6^{\mathrm{a}}$ & $\ldots$ \\
\hline HIP 5104 & 8 & $+2 /-2$ \\
\hline HIP 45158 & 9 & $+3 /-1$ \\
\hline HIP 45033 & 10 & $+2 /-2$ \\
\hline HIP 54048 & 10 & $+2 /-1.5$ \\
\hline HD 84686 & 10 & $+4 /-2$ \\
\hline HIP 72631 & 12 & $+3 /-2$ \\
\hline HIP 13339 & 12 & $+5 /-2$ \\
\hline HIP 19740 & 14 & $+4 /-2$ \\
\hline HIP 3031 & 15 & $+5 /-5$ \\
\hline HIP 71837 & 15 & $+3 /-3$ \\
\hline HIP 8404 & 15 & $+4 /-2$ \\
\hline HIP 78990 & 15 & $+3 /-2$ \\
\hline HIP 94779 & 15 & $+3 /-2$ \\
\hline HIP 44154 & 16 & $+3 /-4$ \\
\hline HIP 94598 & 17 & $+5 /-3$ \\
\hline HIP 98587 & 18 & $+5 /-2$ \\
\hline HIP 100274 & 20 & $+3 /-4$ \\
\hline HIP 75823 & 20 & $+8 /-5$ \\
\hline HIP 60140 & 20 & $+8 /-4$ \\
\hline HIP 103004 & 20 & $+8 /-5$ \\
\hline HIP 56194 & 20 & $+7 /-5$ \\
\hline HIP 90906 & 20 & $+5 /-5$ \\
\hline $\mathrm{BD}+542710$ & 20 & $+5 /-3$ \\
\hline HIP 113610 & 20 & $+7 /-4$ \\
\hline HIP 80309 & 20 & $+5 /-3$ \\
\hline HIP 92827 & 22 & $+8 /-4$ \\
\hline HIP 4197 & 22 & $+4 /-3$ \\
\hline HIP 118209 & 22 & $+7 /-3$ \\
\hline HIP 60485 & 25 & $+5 /-5$ \\
\hline HIP 89008 & 25 & $+7 /-5$ \\
\hline HIP 85715 & 25 & $+5 /-5$ \\
\hline HIP 66892 & 30 & $>+10$ \\
\hline HIP 62325 & 30 & $>+10$ \\
\hline HIP 19611 & 30 & $>+10$ \\
\hline HIP 91985 & 30 & $>+10$ \\
\hline HIP 51179 & 30 & $>+10$ \\
\hline HIP 103734 & 30 & $>+10$ \\
\hline HIP 27280 & 30 & $>+10$ \\
\hline
\end{tabular}

Note. ${ }^{a}$ Taken from Gratton et al. (2000).

But interstellar medium (ISM) dust extinction is patchy and varies considerably toward different sightlines, and many of our stars have poorly constrained distances. Therefore, we estimated their reddening in three different ways. In all cases, we adopted a typical Local Bubble radius of $75 \mathrm{pc}$ and assumed no reddening for stars estimated to be within that distance (e.g., Henry et al. 2000).

For our first reddening estimate, we have applied an isotropic reddening correction for the stars have distances $75 \mathrm{pc}<d \leqslant$ $300 \mathrm{pc}$, adopting $A_{v}=0.8 \mathrm{mag} \mathrm{kpc}^{-1}$ (Henry et al. 2000). The extinction values of eight stars with distances $d>300 \mathrm{pc}$ were obtained from the NASA/IPAC Extragalactic Database $(\mathrm{NED})^{13}$ extinction calculator. Zero reddening was assumed for the stars with high Galactic latitudes, $|b| \geqslant 50^{\circ}$ (e.g., Sandage 1972). For the second and third reddening estimates,

\footnotetext{
$13 \mathrm{http} / / /$ ned.ipac.caltech.edu/
}

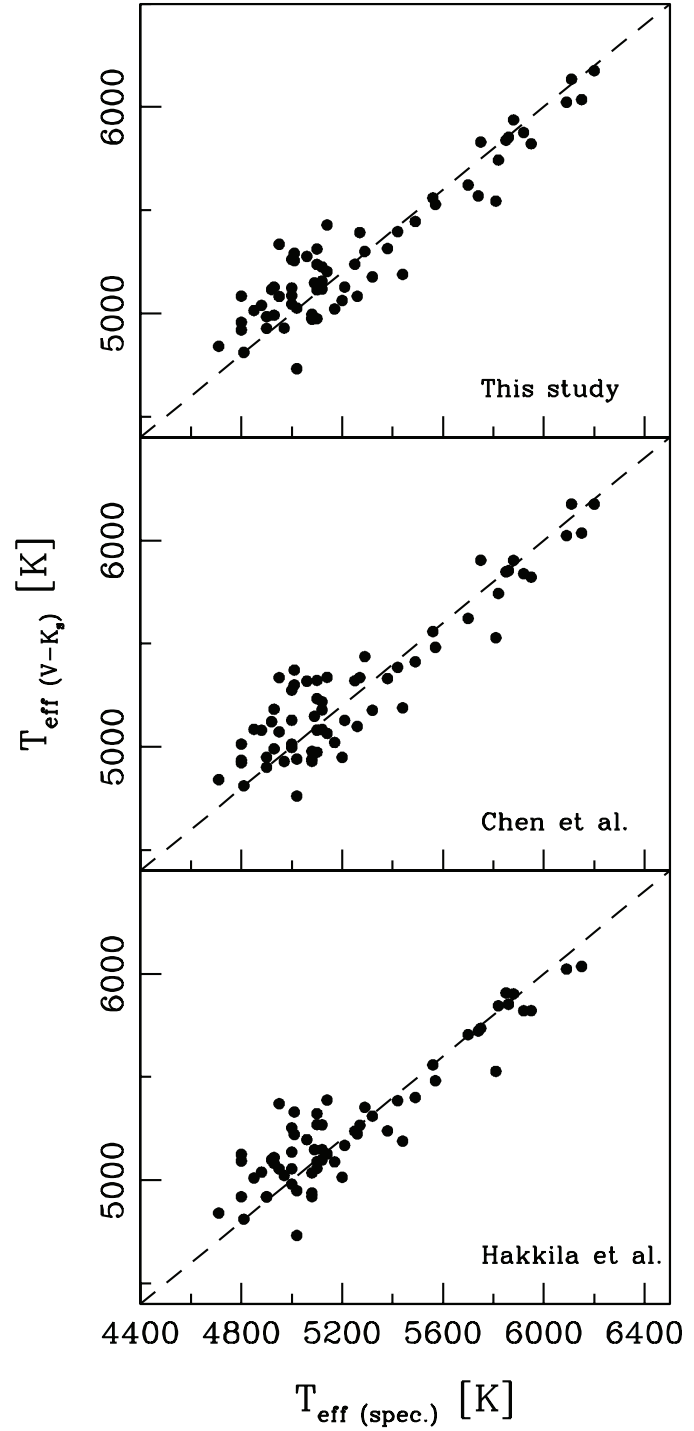

Figure 5. Comparison of spectroscopic $T_{\text {eff }}$ (spec.) with photometric $T_{\text {eff }}$ $\left(V-K_{s}\right)$, adopting the three different reddening estimates discussed in Section 3.2.

we employed the methods described by Chen et al. (1998) and Hakkila et al. (1997). Our computations with the latter method used Hakkila's EXTINCT code ${ }^{14}$ which takes into account various interstellar extinction correction methods from several studies (see Hakkila et al. 1997 for more details).

We applied each of these reddening estimates to the observed $V-K$ colors, computed photometric temperatures from the reddening-corrected $V-K$ colors using the Ramírez \& Meléndez (2005) formula, and correlated them with our spectroscopic temperatures as shown in Figure 5. Inspection of this figure clearly suggests that the two $T_{\text {eff }}$ scales are well correlated, and that various reddening assumptions produce little substantive variations in the temperature correlations. Therefore, we have adopted our $E(B-V)$ values for reddening for the entire sample.

\section{ABUNDANCE DETERMINATIONS}

Using the model atmospheres given in Table 3, we determined abundances of several elements in our program stars. We report

\footnotetext{
${ }^{14}$ Available at http://ascl.net/extinct.html.
} 

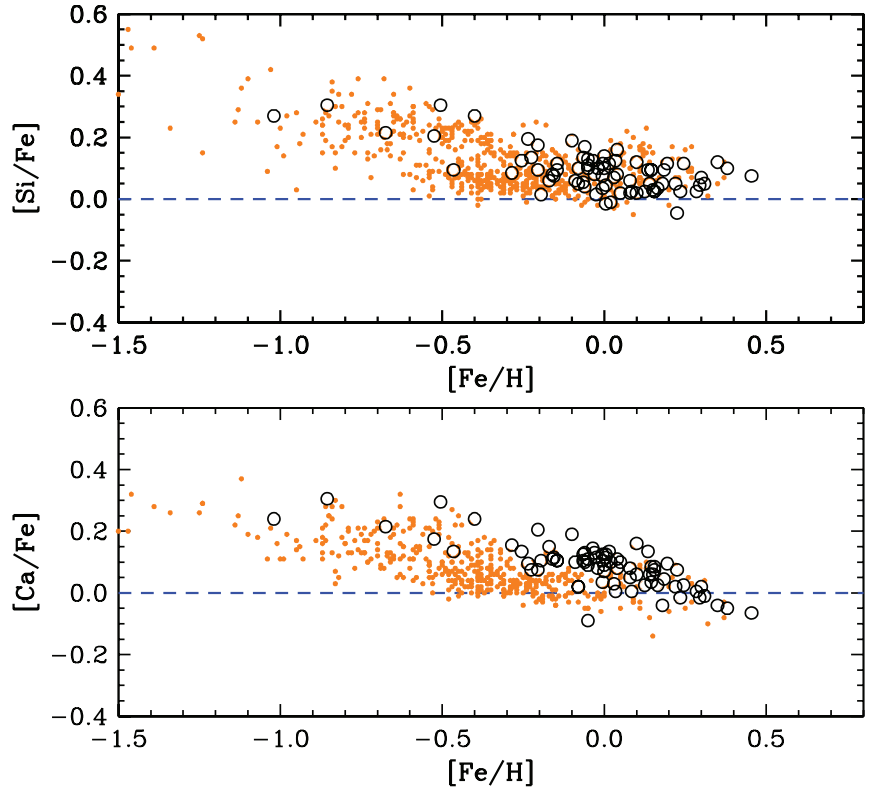

Figure 6. Relative $\mathrm{Si}$ and $\mathrm{Ca}$ abundances in our program stars (black open circles) and in stars in the literature (orange dots). All results are based on lines of Si I and $\mathrm{Ca}$ I species. The data from the literature are adopted without adjustment from Tautvaišienè et al. (2001), Bensby et al. (2003), Reddy et al. (2003, 2006), and Mishenina et al. (2006).

(A color version of this figure is available in the online journal.)

these as relative abundance ratios $[\mathrm{X} / \mathrm{H}]$ in Table 7 . Whenever possible, we computed the abundances from line EWs. For complex transitions, those that have significant hyperfine and isotopic substructure, and those that have significant line blending issues, we resorted to synthetic/observed spectrum matches to determine the abundances. We were especially interested in three element groups that could help constrain the Galactic population memberships of our stars and verify their evolutionary states. In the following subsections we consider in turn the $\alpha$, the neutron-capture, and the proton-capture element groups.

\section{1. $\alpha$-elements: Silicon and Calcium}

The abundances of $\alpha$-elements exhibit the same behavior in giant and dwarf stars of solar metallicity $([\alpha / \mathrm{Fe}] \sim 0$; Soubiran $\&$ Girard 2005; Mishenina et al. 2006). In thick-disk MS and subgiant stars, the $\alpha$ elements typically become overabundant as metallicity decreases, reaching $[\alpha / \mathrm{Fe}] \sim+0.3$ at $[\mathrm{Fe} / \mathrm{H}] \sim$ -1 (e.g., Tautvaišiené et al. 2001; Reddy et al. 2006). The temperatures and gravities of these prior large samples are $5000 \mathrm{~K} \lesssim T_{\text {eff }} \lesssim 6500 \mathrm{~K}$ and $3.4 \lesssim \log g \lesssim 4.7$. Less systematic abundance trend information is available for thin/thick-disk giant stars, which in our case cover the approximate parameter range $4800 \mathrm{~K} \lesssim T_{\text {eff }} \lesssim 5600 \mathrm{~K}$ and $2.2 \lesssim \log g \lesssim 4.0$.

The easily observable $\alpha$ elements are $\mathrm{Mg}, \mathrm{Si}$, and $\mathrm{Ca}$. Often $\mathrm{Ti}$ is grouped with the other $\alpha$ due to its similar abundance behavior with metallicity. However, Ti is not a pure $\alpha$ element because its dominant isotope is ${ }^{48} \mathrm{Ti}_{22}$, which is not an even multiple of $\alpha$ particles. Here we concentrated on $\mathrm{Si}$ and $\mathrm{Ca}$ abundances, since they have large numbers of transitions with a range of line strengths in the yellow-red spectral region (most $\mathrm{Mg}$ I lines are very strong in our $\mathrm{G}-\mathrm{K}$ giant stars). We generally used about $9 \mathrm{Si}$ I and $15 \mathrm{Ca}$ I lines, and derived abundances from their EWs.

We compare our $\mathrm{Si}$ and $\mathrm{Ca}$ abundances with published values in Figure 6. The literature data are taken from several studies that are given in the figure caption: Tautvaišienè et al. (2001), Reddy et al. (2003, 2006), Bensby et al. (2003) and Mishenina et al. (2006). Our sample has a wide metallicity range $-1.0 \leqslant$ $[\mathrm{Fe} / \mathrm{H}]<+0.5$ and it is clear that the $\alpha$ elements behave similarly in our cooler giants as they do in warmer MS stars and subgiants. Even though slight scaling differences $(<0.1 \mathrm{dex})$ naturally result from different solar abundances, different line choices, and different oscillator strengths adopted here compared with previous studies, our results are generally in good agreement with the literature. At a given $[\mathrm{Fe} / \mathrm{H}]$ metallicity, our mean $[\mathrm{Ca} / \mathrm{Fe}]$ values are typically within 0.05 dex of literature values, and $[\mathrm{Si} / \mathrm{Fe}]$ are within $0.01 \mathrm{dex}$.

\subsection{Neutron-capture Elements}

Among thin- and thick-disk stars, those elements whose solarsystem origin is due chiefly to "slow" neutron bombardment reactions (the $s$-process; e.g., $\mathrm{Sr}, \mathrm{Y}, \mathrm{Zr}, \mathrm{Ba}, \mathrm{La}$, and $\mathrm{Ce}$ ) generally exhibit their solar abundance ratios $([\mathrm{X} / \mathrm{Fe}] \sim 0)$ throughout metallicity regime $-1.0 \lesssim[\mathrm{Fe} / \mathrm{H}] \lesssim+0.2$. But those elements that are products of "rapid"-blast neutron-capture events (the $r$-process; Eu, Gd, Dy) tend to increase in relative abundance with decreasing metallicity. The most easily observed $r$-process element is $\mathrm{Eu}$, and its mean abundances reaches $[\mathrm{Eu} / \mathrm{Fe}] \sim+0.4$ at $[\mathrm{Fe} / \mathrm{H}] \sim-1$. A good summary of the disk $s$ - and $r$-process abundance trends can be seen in Figure 17 of Reddy et al. (2006). Additionally, Simmerer et al. (2004) have shown that a distinct kinematic signature in disk neutron-capture elements: stars with larger space motions have often much lower $s$-process abundances than $r$-process ones (see their Figure 12).

There are not a lot of useful neutron-capture element $(Z>30)$ transitions in the yellow-red spectral region. We considered just La (75\% s-process origin in solar-system material; e.g., Sneden et al. 2008 and references therein), and $\mathrm{Eu}$ (97\% $r$-process).

La II. Transitions at 6262.2 and $6390.5 \AA$ can be detected in nearly all of our stars. These lines have well-determined transition probabilities and hyperfine structure parameters (Lawler et al. 2001a; see also Ivans et al. 2006 for complete substructure line lists for these transitions). The sole naturally occurring isotope of this element is ${ }^{139} \mathrm{La}$. Because of the complexity of these La II transitions we used synthetic spectrum analyses to determine the abundances. Notable contaminants to the La features are $\mathrm{CN}$ red-system lines, but in most cases the $\mathrm{CN}$ strengths were small and did not materially affect the derived abundances.

Eu II. Transitions at 6645.1 and 7217.5 ̊ were used in the Eu abundance analyses. The transitions are very complex because $\mathrm{Eu}$ has both hyperfine and isotopic substructure. There are two Eu stable isotopes, ${ }^{151} \mathrm{Eu}(48 \%$ of the total solar-system $\mathrm{Eu}$ abundance) and ${ }^{153} \mathrm{Eu}(52 \%)$. There is little difference in $\mathrm{Eu}$ isotopic ratios generated in $r$-process and $s$-process environments. Spectroscopic studies of Eu II in extremely metalpoor, Eu-enhanced stars suggest that the ${ }^{151} \mathrm{Eu}$ fraction is $\approx 50 \%$ for $r$-rich stars (Sneden et al. 2002; Aoki et al. 2003a; Roederer et al. 2008) and $\approx 57 \%$ for $s$-rich stars (Aoki et al. 2003b). Therefore in our Eu syntheses we adopted the solar-system isotopic ratios. The basic laboratory analyses of these lines were published by Lawler et al. (2001b); full hyperfine/isotopic substructure lists are in Ivans et al. (2006).

The abundance variations for La II and Eu II as a function of metallicity are given in Figure 7. The observed trends (no apparent change in $[\mathrm{La} / \mathrm{Fe}]$, increasing $[\mathrm{Eu} / \mathrm{Fe}]$ at lower metallicities) are in complete agreement with previous results cited above. The neutron-capture element abundances, combined with those 

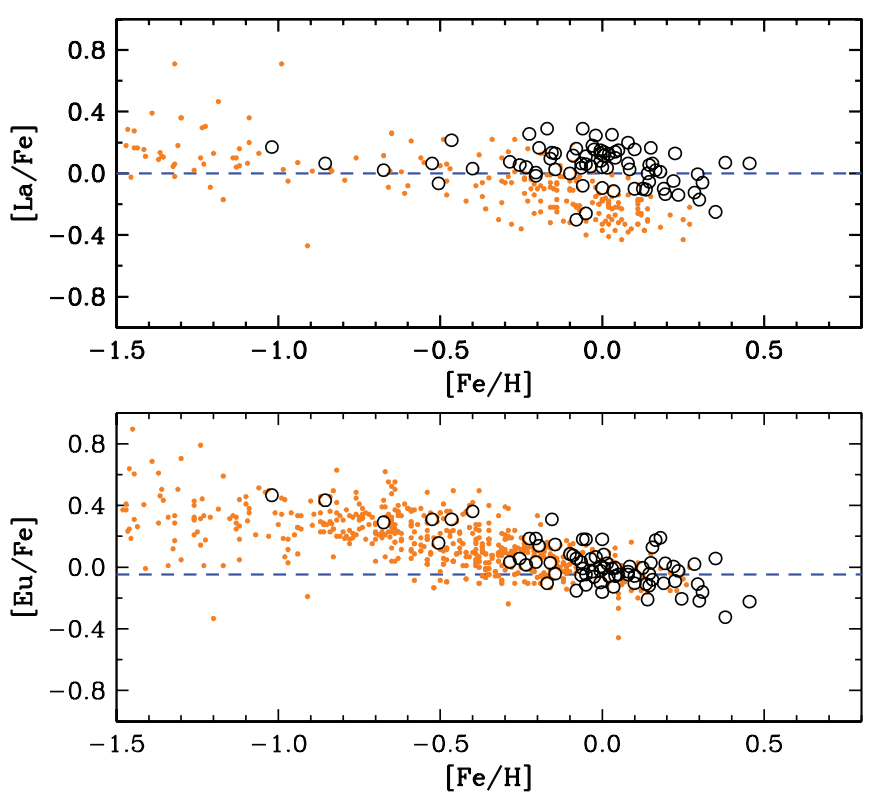

Figure 7. Relative $\mathrm{La}$ and $\mathrm{Eu}$ abundances in our program stars (black open circles) and in stars in the literature (orange dots). All results are based on lines of La II and Eu II species. The data from the literature are adopted without adjustment from Tautvaišiené et al. (2001), Bensby et al. (2003), Venn et al (2004), Simmerer et al. (2004), Reddy et al. (2003, 2006), and Mishenina et al. (2007).

(A color version of this figure is available in the online journal.)

of the $\alpha$ elements clearly provide Galactic population indicators for our stars.

\subsection{The Proton-capture Abundances}

Evolved Population I solar-metallicity stars show evidence of convective envelope mixing; their observed light elements $\mathrm{Li}, \mathrm{C}, \mathrm{N}$, and $\mathrm{O}$ clearly have been altered via interior synthesis in $p-p$ and $\mathrm{CNO}$ cycles. The original $\mathrm{C}$ abundance drops by a factor of about two, the $\mathrm{N}$ abundance rises by comparable amounts, and the carbon isotopic ratio drops to values usually between 15 and 30 (e.g., Lambert \& Ries 1981). Mildly metalpoor, high-velocity stars (thick disk, labeled old disk in early papers) of about a solar mass have lower ${ }^{12} \mathrm{C} /{ }^{13} \mathrm{C}$ ratios but less evidence for depleted C and enhanced N (e.g., Cottrell \& Sneden 1986). Low-mass giant stars of the Galactic halo often display more dramatic ${ }^{12} \mathrm{C} \rightarrow{ }^{13} \mathrm{C}$ and $\mathrm{C} \rightarrow \mathrm{N}$ conversions (e.g., Gratton et al. 2000). Thus the CNO abundances of evolved stars can yield information on their internal evolutions and population memberships.

Since stars easily destroy $\mathrm{Li}$ in relatively low temperature proton-capture reactions, $\mathrm{Li}$ abundances or upper limit estimates in metal-rich stars provide additional information. The presence of $\mathrm{Li}$ in the spectrum of an evolving star usually suggests that envelope convection has not yet developed to a point where dredge-down of original surface Li has effectively cleaned it from the star's envelope. The absence of Li can indicate (but not always) that other proton-capture products may have been dredged up to the surface. This straightforward interpretation is complicated by the relatively rare phenomenon of Li-rich giants (e.g., Charbonnel \& Balachandran 2000; Kumar et al. 2011, and references therein). This increases the importance of searching for this element in the spectrum of any evolved star.

Unfortunately, many atomic and molecular transitions that are CNOLi abundance indicators have significant detection and/or

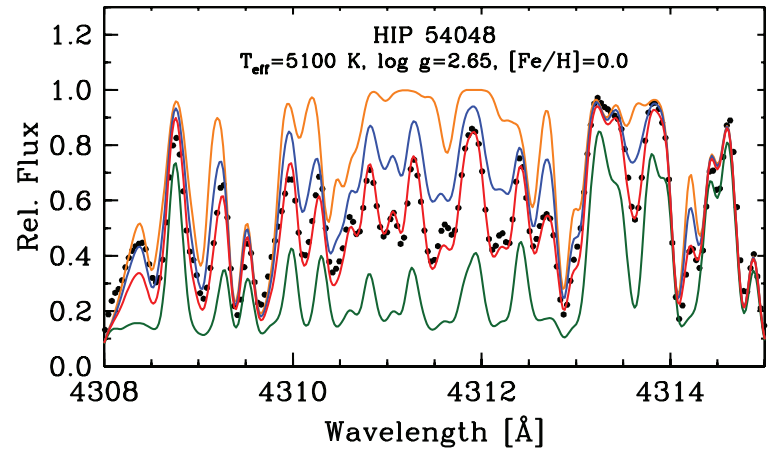

Figure 8. Comparison of synthetic spectrum and observed spectrum of HIP 54048 for part of the $\mathrm{CH} G$-band region. Best fit is illustrated by a red solid line for $\log \epsilon(\mathrm{C})=7.79$. Other fits represented by yellow, blue, and green solid lines are given for $\log \epsilon(\mathrm{C})=2.79,7.34$, and 8.69, respectively.

(A color version of this figure is available in the online journal.)

analytical issues. Here we describe the transitions that we used, and our atomic/molecular parameter choices.

CI. High-excitation $(\chi \gtrsim 7.7 \mathrm{eV})$ lines of this species are strong in the warmest candidate stars but weaken with decreasing $T_{\text {eff }}$. We adopted the transition probabilities for these lines recommended in the NIST Atomic Spectra Database (Ralchenko et al. 2011). ${ }^{15}$ However, the $\mathrm{C}_{\mathrm{I}}$ lines decrease in strength so quickly with decreasing $T_{\text {eff }}$ that often only a couple of them could be used for $\mathrm{C}$ abundances in our stars.

CH G band. We also employed the $\mathrm{CH} X^{2} \Pi-A^{2} \Delta$ " $G$ band" to derive $\mathrm{C}$ abundances via spectrum syntheses. Significant $G$-band absorption occurs in the 4200-4400 $\AA$ spectral range, and we estimated $\mathrm{C}$ abundances from three regions

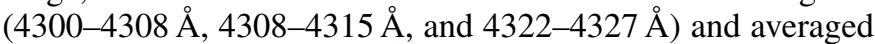
the results. The $4308-4315 \AA$ region proved to be the most reliable $\mathrm{C}$ abundance indicator, due to greater atomic-line contamination in the other two spectral regions. The synthetic spectrum line list was formed with ${ }^{12} \mathrm{CH}$ and ${ }^{13} \mathrm{CH}$ lines from $\mathrm{B}$. Plez (2009, private communication; see, e.g., Hill et al. 2002; Plez \& Cohen 2005) and atomic lines from the Kurucz (2011) compendium. The $\mathrm{CH}$ lines are plentiful and relatively strong throughout the atmospheric parameter domain of our program stars. As an example of the $\mathrm{CH}$ observed/synthetic spectrum matches, we show in Figure 8 the 4308-4315 $\AA$ spectral region in a typical program star. Generally more reliable $\mathrm{C}$ abundances were obtained from the $\mathrm{CH}$-band syntheses than those from the C I EW measurements, especially among the cooler $\left(T_{\text {eff }}<\right.$ $5500 \mathrm{~K})$ stars.

CN. We used synthetic spectrum calculations of ${ }^{12} \mathrm{CN}$ and ${ }^{13} \mathrm{CN} A^{2} \Pi-X^{2} \Sigma$ red-system lines in the $7995-8040 \AA$ region to determine $\mathrm{N}$ abundances and ${ }^{12} \mathrm{C} /{ }^{13} \mathrm{C}$ ratios. The synthesis line lists were taken from Kurucz (2011). A triplet of ${ }^{13} \mathrm{CN}$ lines near $8004.7 \AA$ was the primary ${ }^{12} \mathrm{C} /{ }^{13} \mathrm{C}$ indicator, but several other, usually weaker, features (e.g., at 8007.9, 8010.4, and $8011.2 \AA$ ) were used for confirmation. In Figure 9, we give an example of a synthetic spectrum fit to the ${ }^{12} \mathrm{CN}$ and ${ }^{13} \mathrm{CN}$ features in the $8002.5-8011.5 \AA$ region. Derived ${ }^{12} \mathrm{C} /{ }^{13} \mathrm{C}$ ratios for our program stars are given in Table 5. $\mathrm{C}-\mathrm{N}-\mathrm{O}$ abundances are bound to each other through molecular equilibrium, which becomes more important toward lower temperatures. The accuracy of $\mathrm{N}$ abundances especially depends on the accuracy of $\mathrm{C}$ abundances through $\mathrm{CN}$ formation. Note that our line list produced a systematic offset of $\sim 0.15$ in the solar N abundance (Table 4) compared to Asplund et al. (2009).

\footnotetext{
15 Available at http://physics.nist.gov/asd3.
} 


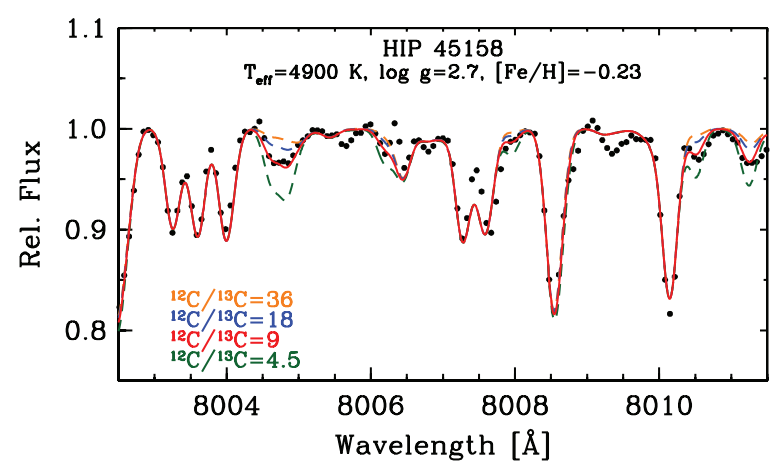

Figure 9. Comparison of synthetic spectrum and observed spectrum of HIP 45158 for the observed ${ }^{13} \mathrm{CN}$ feature around $8004 \AA$. Best fit is illustrated by a red solid line for ${ }^{12} \mathrm{C} /{ }^{13} \mathrm{C}=9$. Other trials are also shown for ${ }^{12} \mathrm{C} /{ }^{13} \mathrm{C}$ values of 36,18, and 4.5 (dashed lines).

(A color version of this figure is available in the online journal.)

This would suggest that perhaps we have an offset of about +0.15 dex in our $\mathrm{N}$ abundances.

$[\mathbf{O} \mathbf{I}]$. There are two ground-state forbidden transitions upon which are based most of the $\mathrm{O}$ abundances in red giant stars. We analyzed only the $6300.3 \AA$; its companion at $6363.8 \AA$ is much weaker and suffers from large amounts of $\mathrm{CN}$ contamination. The $6300 \AA$ line has a very accurate transition probability (Allende Prieto et al. 2001 and references therein). The $6300 \AA$ [O I] line is blended with an Ni I line at $6300.34 \AA$; see Allende Prieto et al. One needs to remove its contribution carefully during the modeling of the [O I] spectral region around $6300 \AA$. Of some importance is the Ni I oscillator strength; we use the value given by Johansson et al. (2003).

$\mathbf{O}$ I. In the spectra of our warmer stars the forbidden line is too weak to yield reliable abundances, so we also used the oftenanalyzed very high excitation $7770 \AA$ triplet. For these lines we adopted $g f$-values from the NIST database (Ralchenko et al. 2011). Unfortunately, it is well known that the O I triplet lines are subject to NLTE effects, in the sense that their LTE-based abundances are always too large $(\lesssim 0.2$ dex; Gratton et al. 1999; Bensby et al. 2004) compared to abundances from the forbidden lines. In order to correct for the NLTE effects, we followed a similar approach to the NLTE-correction method of Bensby et al. (2004). That is, we applied a "robust regression" analysis and derived the following equation for $\left[\mathrm{O}_{6300} / \mathrm{O}_{7774}\right]_{\text {cor }}$ as a function of $T_{\text {eff }}, \log g$, and $[\mathrm{Fe} / \mathrm{H}]$ :

$$
\begin{aligned}
{\left[\mathrm{O}_{6300} / \mathrm{O}_{7774}\right]_{\text {cor }}=} & -0.293( \pm 0.027) \\
& +0.429( \pm 0.839) \cdot \log \left(\frac{T_{\text {eff }}}{T_{\text {eff } \odot}}\right) \\
& -0.232( \pm 0.033) \cdot \log \left(\frac{\log g_{\star}}{\log g_{\odot}}\right) \\
& +0.136( \pm 0.055) \cdot[\mathrm{Fe} / \mathrm{H}] .
\end{aligned}
$$

Here, $\left[\mathrm{O}_{6300} / \mathrm{O}_{7774}\right]_{\text {cor }}$ represents the difference between the oxygen abundances gathered from the $\left[\mathrm{OI}_{6300}\right.$ line and the $[\mathrm{O}]_{7774}$ triplet lines. Then we simply subtracted this difference from $[\mathrm{O} / \mathrm{H}]_{7774}$ abundances in order to obtain NLTE-corrected $[\mathrm{O} / \mathrm{H}]_{7774}^{\mathrm{NLTE}}$ values. In Figure 10, we compare the deviation of the differences between $\left[\mathrm{O}_{6300} / \mathrm{O}_{7774}\right]_{\text {LTE }}$ and $\left[\mathrm{O}_{6300} / \mathrm{O}_{7774}\right]_{\text {NLTE }}$. The mean and the standard deviation of the correlation after correction are 0.05 dex and $\sigma \simeq \pm 0.12 \mathrm{dex}$, respectively.

In Figure 11, we plot our $\mathrm{CNO}$ abundances along with previously reported values, using the same comparison samples.
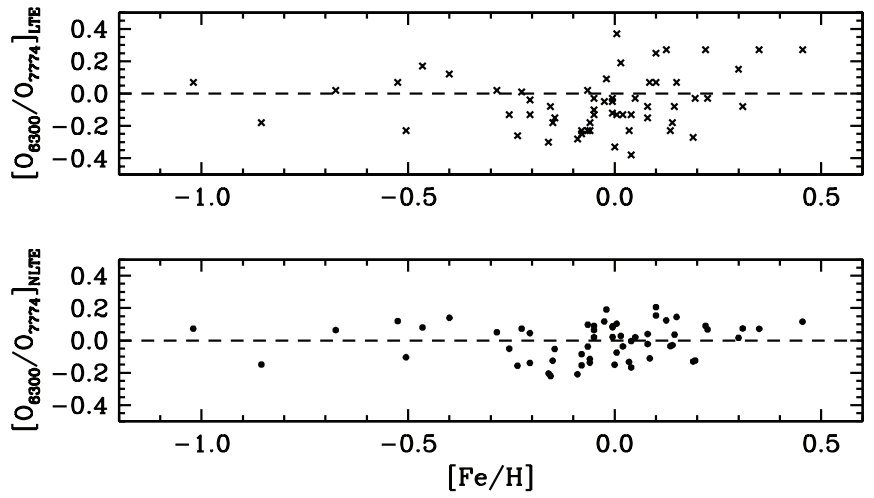

Figure 10. Application of NLTE correction to the OI triplet. Upper panel (crosses) shows before, bottom panel (dots) shows after the correction applied.
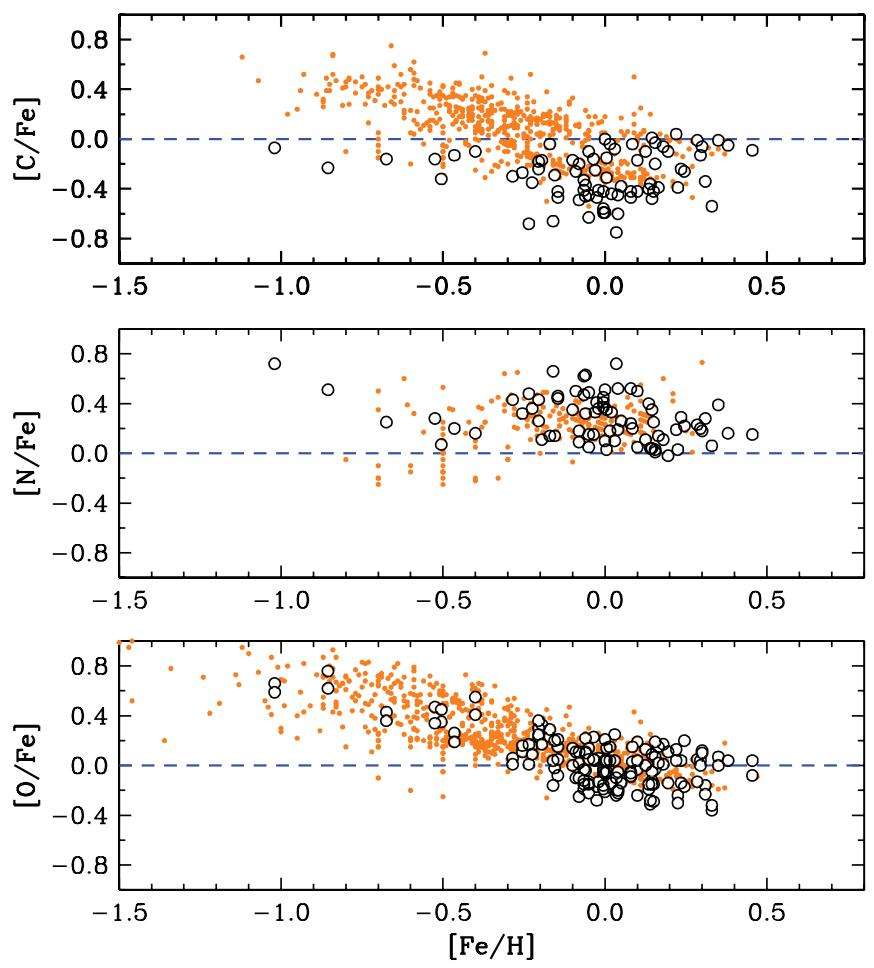

Figure 11. Relative $\mathrm{C}, \mathrm{N}$, and $\mathrm{O}$ abundances in our program stars (black open circles) and in stars in the literature (orange dots). All results are based on lines of $\mathrm{C}_{\mathrm{I}_{\mathrm{CH}}}, \mathrm{N}$ I from CN8020 Åregion and $\mathrm{O}_{6300}$ as well as NLTE-corrected O I from 7774 triplets species. The data from the literature are adopted without adjustment from Lambert \& Ries (1981), Cottrell \& Sneden (1986), Tautvaišienè et al. (2001), Bensby et al. (2003), Reddy et al. (2003, 2006), and Mishenina et al. (2006).

(A color version of this figure is available in the online journal.)

While the derived mean $\mathrm{O}$ and $\mathrm{N}$ abundances of our stars are in good agreement with literature mean values at similar metallicities, our $\mathrm{C}$ abundances are somewhat lower than those from previous studies. But the comparison samples in this case are not the best, because the literature data are dominated by MS and subgiant stars, while our program stars are clearly much more evolved. Therefore in Figure 12 we repeat the exercise of the previous figure, but with two differences. First, we have eliminated the stars of our sample that are probably not chemically mixed (those with $T \gtrsim 5400 \mathrm{~K}$ and/or $\log g \gtrsim 3.5$ ). Second, we added literature CNO abundance results only from studies that concentrated on red giant stars. Our $\mathrm{C}$ abundances are more in accord with those in other samples of evolved giants. 

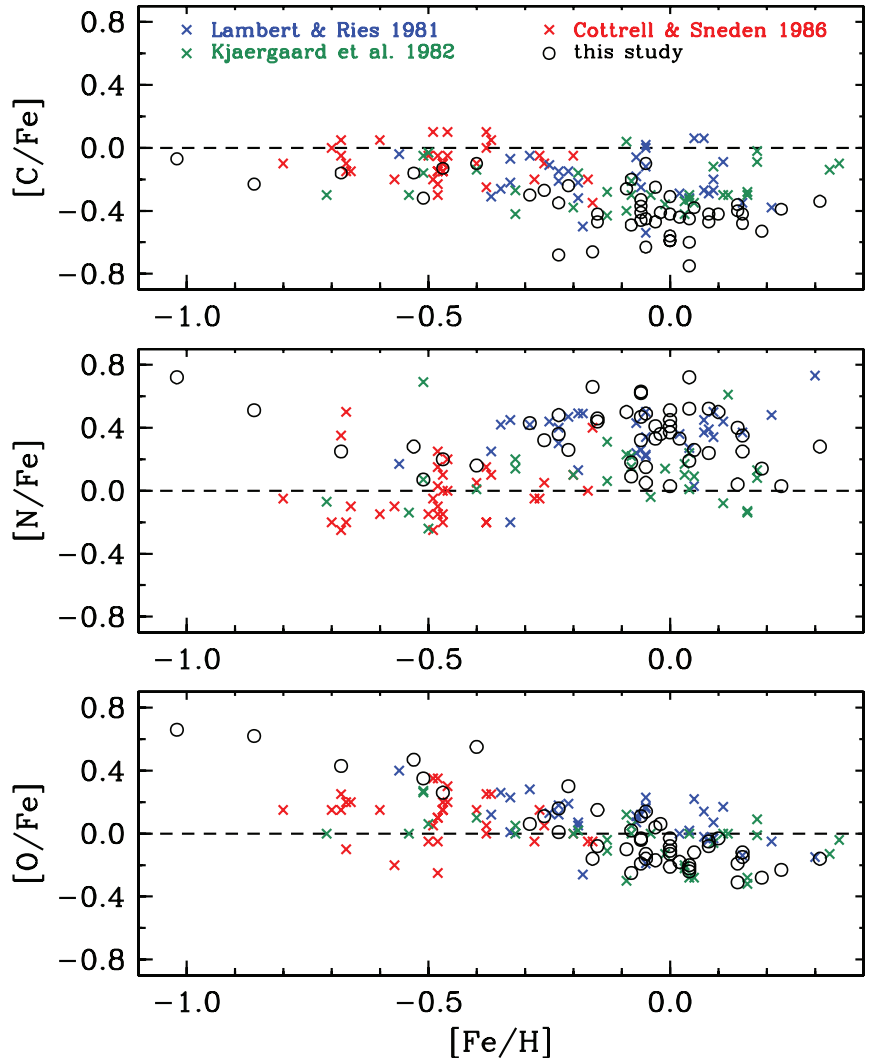

Figure 12. $\mathrm{C}, \mathrm{N}$, and $\mathrm{O}$ abundances in our program stars (open circles) and in samples of evolved (red giant) stars in the literature (crosses). Different colors have been used to denote results from other studies, according to the legend given in the top panel.

(A color version of this figure is available in the online journal.)

Thick-disk giants $([\mathrm{Fe} / \mathrm{H}]<-0.25)$ in general show higher $\mathrm{C}$ and $\mathrm{O}$ abundances than their thin-disk counterparts. The $\mathrm{O}$ enrichment is indicative of larger Type II SN contributions to thick disk than thin-disk stars, combined with no depletion in $\mathrm{ON}$-cycle $\mathrm{H}$-fusion (interior temperatures are too low) in the low-mass thick-disk giants that we observe. The higher $\mathrm{C}$ in thick-disk stars suggests that the lower the metallicity, relatively low mass thick-disk giants have relatively shallow convective envelopes, and dredge-up has failed to reach the interior fusion zones where the $\mathrm{CN}$-cycle has run to completion. This echoes the discussion in Cottrell \& Sneden 1986, who concluded that in their old disk giants, "only the coolest (outer) portions of the CNO-processed hydrogen-burning shell, where ${ }^{12} \mathrm{C}(p, \gamma){ }^{13} \mathrm{C}$ has taken place, have been convectively mixed into outer layers." Theoretical support for this notion was given in Sneden et al. (1986), whose Table 6 gives D. A. VandenBerg's predicted $\mathrm{C} / \mathrm{N}$ ratios from standard evolutionary model stars that have experienced first dredge-up. It is clear that as mass and metallicity decrease, model stars exhibit decreasing surface $\mathrm{C}$ and $\mathrm{N}$ abundance changes.

Li I. Unless the Li abundance is extraordinarily large, only the $6707.8 \AA$ resonance line of the neutral species can be detected in cool stars. This ground-state doublet transition has a welldetermined $g f$ value; see the summary of experimental and theoretical studies in Smith et al. (1998). The transition is complex, with both isotopic and hyperfine substructure. We adopted the parameters given in the line compendium of Kurucz (2011). ${ }^{16}$

\footnotetext{
16 http://kurucz.harvard.edu/linelists.html
}

Table 6

Li Abundances

\begin{tabular}{|c|c|c|c|c|c|}
\hline Stars & $T_{\text {eff }}$ & $\log g$ & {$[\mathrm{Fe} / \mathrm{H}]$} & $\log \epsilon(\mathrm{Li})$ & ${ }^{12} \mathrm{C} /{ }^{13} \mathrm{C}$ \\
\hline HIP 45033 & 5120 & 2.75 & 0.02 & 0.93 & 10 \\
\hline HIP 94598 & 5010 & 2.45 & -0.05 & 0.96 & 17 \\
\hline $\mathrm{BD}+542710$ & 4840 & 2.20 & 0.04 & 0.56 & 20 \\
\hline HIP 92827 & 4850 & 2.70 & 0.04 & 0.74 & 22 \\
\hline HIP 4197 & 4800 & 2.50 & -0.23 & 0.60 & 22 \\
\hline HIP 89008 & 5000 & 2.55 & 0.14 & 1.04 & 25 \\
\hline HIP 103734 & 5060 & 2.65 & 0.31 & 1.34 & $>30$ \\
\hline HIP 19611 & 5080 & 2.90 & 0.00 & 1.56 & $>30$ \\
\hline HIP 51179 & 5020 & 2.80 & -0.15 & 0.75 & $>30$ \\
\hline HIP 476 & 5140 & 2.80 & -0.03 & 0.91 & $\ldots$ \\
\hline HIP 60873 & 5080 & 3.25 & -0.47 & 0.81 & $\ldots$ \\
\hline BD+45 2032 & 5420 & 3.70 & -0.16 & 1.54 & $\ldots$ \\
\hline HIP 82014 & 5490 & 3.90 & 0.13 & 2.04 & $\ldots$ \\
\hline HIP 80543 & 5920 & 3.65 & 0.15 & 3.26 & $\ldots$ \\
\hline HIP 11924 & 5850 & 4.00 & 0.30 & 1.70 & $\ldots$ \\
\hline HD 96780 & 6200 & 4.00 & 0.01 & 2.81 & $\ldots$ \\
\hline HD 141770 & 5650 & 4.05 & 0.09 & 2.26 & $\ldots$ \\
\hline $\mathrm{BD}+412221$ & 5810 & 4.15 & 0.22 & 2.44 & $\ldots$ \\
\hline HIP 46325 & 5650 & 4.15 & 0.46 & 1.92 & $\ldots$ \\
\hline TYC 3720-324-1 & 5960 & 4.20 & 0.38 & 2.69 & $\ldots$ \\
\hline HD 101014 & 5880 & 4.20 & 0.30 & 2.20 & $\ldots$ \\
\hline BD+32 2190 & 5860 & 4.25 & 0.29 & 1.59 & $\ldots$ \\
\hline HIP 58269 & 5820 & 4.30 & 0.35 & 2.06 & $\ldots$ \\
\hline $\mathrm{BD}+312565$ & 6150 & 4.30 & 0.03 & 2.57 & $\ldots$ \\
\hline HD 242647 & 5890 & 4.30 & -0.20 & 2.09 & $\ldots$ \\
\hline HIP 65900 & 6090 & 4.40 & 0.15 & 2.72 & $\ldots$ \\
\hline HD 166310 & 5480 & 4.60 & 0.17 & 2.03 & $\ldots$ \\
\hline
\end{tabular}

We were able to measure Li I abundances for some of our targets (see Table 6). Since Li line strength is a severe function of $T_{\text {eff }}$, for stars with undetectable Li the approximate abundance upper limits as guidelines for interpretation are: for stars with $\log g \geqslant 3.5$ and $T \geqslant 5500, \log \epsilon(\mathrm{Li})<1$, and for those with $\log g<3.0$ and $T \leqslant 5100, \log \epsilon(\mathrm{Li})<+0.5$ (with the limit of course decreasing toward lower temperatures). The $\mathrm{Li}$ abundances decrease as $T_{\text {eff }}$ and $\log g$ decrease. This is as expected in normal stellar evolution: during the path from MS to red giant phases, the deepening convective envelopes bring Li down from the surface layers where it can be destroyed easily at relatively low fusion-zone temperatures. Program stars with higher surface Li abundances have undetectable ${ }^{13} \mathrm{C}$, consistent with their chemically un-evolved status. Six stars of our sample have low ${ }^{12} \mathrm{C} /{ }^{13} \mathrm{C}$ values, but detectable Li I lines; these all appear to be plausible RHB and RC candidates (see Table 7). For these stars, $\langle\log \epsilon(\mathrm{Li})\rangle \sim 0.8$. Standard evolution models (e.g., those without extra-mixing mechanisms seeming needed for Population II giants) predict surface Li depletion by factors up to about 30 (Iben 1967a). If a star begins MS life with the present ISM Li abundance $(\log \epsilon(\mathrm{Li}) \sim+3.0$; e.g., Grevesse \& Sauval 1998) then through ordinary stellar evolution it should exhibit $\log \epsilon(\mathrm{Li}) \lesssim+1.5$. None of our evolved program stars have $\mathrm{Li}$ abundances near this value, indicating either that their natal $\mathrm{Li}$ contents were not as high as the present ISM value, or that extra-mixing during their lives destroyed Li beyond the standard predictions.

\section{KINEMATICS}

Among the overall sample of 76 stars, we have distance information for 58 of them to facilitate a kinematical investigation. In order to calculate stellar space velocities, we have collected parallaxes and proper motions, as well as their errors, from van 
Table 7

Abundances

\begin{tabular}{|c|c|c|c|c|c|c|c|c|c|c|}
\hline Stars & [Si I/Fe] & {$[\mathrm{Ca} \mathrm{I} / \mathrm{Fe}]$} & {$[\mathrm{C} / \mathrm{Fe}]_{\mathrm{CH}}$} & {$[\mathrm{N} / \mathrm{Fe}]_{\mathrm{CN}}$} & {$[\mathrm{O}$ I/Fe] } & {$[\mathrm{O} / \mathrm{I} / \mathrm{Fe}]_{\text {NLTE }}$} & {$[\mathrm{La} \mathrm{II} / \mathrm{Fe}]$} & {$[\mathrm{Eu}$ II $/ \mathrm{Fe}]$} & ${ }^{12} \mathrm{C} /{ }^{13} \mathrm{C}$ & Evol. Phase \\
\hline \multicolumn{11}{|c|}{ Thick disk } \\
\hline HIP 3031 & 0.21 & 0.18 & -0.16 & 0.28 & 0.47 & 0.34 & 0.07 & 0.38 & 15 & RHB \\
\hline HIP 4960 & 0.27 & 0.24 & -0.07 & 0.72 & 0.66 & 0.59 & 0.17 & 0.54 & 6 & RHB \\
\hline HIP 5104 & 0.31 & 0.31 & -0.23 & 0.51 & 0.62 & 0.76 & 0.07 & 0.51 & 8 & RHB \\
\hline HIP 45412 & 0.22 & 0.22 & -0.16 & 0.25 & 0.43 & 0.36 & 0.02 & 0.36 & 5 & RHB \\
\hline HIP 58269 & 0.12 & -0.04 & -0.01 & 0.39 & 0.07 & 0.00 & -0.25 & 0.11 & $\ldots$ & MS/SG \\
\hline HIP 62325 & 0.17 & 0.10 & -0.46 & 0.32 & -0.04 & 0.10 & -0.08 & 0.04 & 30 & RGB \\
\hline HIP 66892 & 0.10 & 0.08 & -0.41 & 0.36 & 0.06 & -0.13 & 0.25 & 0.12 & 30 & - \\
\hline HIP 71837 & 0.11 & 0.11 & -0.42 & 0.44 & 0.15 & 0.21 & 0.02 & 0.21 & 15 & $\mathrm{RC} / \mathrm{RHB}$ \\
\hline HIP 72631 & 0.18 & 0.08 & -0.24 & 0.26 & 0.30 & 0.25 & -0.01 & 0.25 & 12 & $\mathrm{RC}$ \\
\hline HIP 115839 & 0.27 & 0.24 & -0.10 & 0.16 & 0.55 & 0.41 & 0.03 & 0.43 & 5 & RHB \\
\hline BD+27 2057 & 0.31 & 0.30 & -0.32 & 0.07 & 0.35 & 0.45 & -0.07 & 0.22 & 5 & $\mathrm{RHB} / \mathrm{RC}$ \\
\hline \multicolumn{11}{|c|}{ Thin/thick disk } \\
\hline HIP 11924 & 0.07 & 0.02 & -0.06 & 0.18 & 0.12 & 0.10 & -0.17 & -0.18 & $\ldots$ & $\mathrm{MS} / \mathrm{SG}$ \\
\hline HIP 46325 & 0.08 & -0.07 & -0.09 & 0.15 & 0.04 & -0.08 & 0.06 & -0.19 & $\ldots$ & $\mathrm{MS} / \mathrm{SG}$ \\
\hline HIP 57535 & 0.12 & 0.06 & -0.17 & 0.05 & 0.19 & 0.04 & -0.10 & -0.01 & $\ldots$ & SG \\
\hline HIP 60873 & 0.09 & 0.14 & -0.13 & 0.20 & 0.26 & 0.19 & 0.22 & 0.38 & $\ldots$ & RGB \\
\hline HIP 70341 & 0.10 & 0.07 & 0.00 & 0.10 & $\ldots$ & 0.21 & 0.04 & 0.24 & $\ldots$ & MS/SG \\
\hline HIP 70344 & 0.13 & 0.15 & -0.16 & 0.15 & $\ldots$ & 0.23 & 0.05 & 0.11 & $\ldots$ & SG \\
\hline HIP 75823 & 0.09 & 0.16 & -0.30 & 0.43 & 0.06 & 0.01 & 0.08 & 0.09 & 20 & RHB \\
\hline HIP 82014 & 0.03 & 0.02 & -0.10 & 0.11 & 0.13 & 0.00 & -0.10 & 0.04 & $\ldots$ & SG \\
\hline HIP 98587 & 0.08 & 0.11 & -0.66 & 0.66 & -0.16 & 0.04 & 0.10 & 0.08 & 18 & $\mathrm{RC} / \mathrm{eAGB}$ \\
\hline \multicolumn{11}{|c|}{ Thin disk } \\
\hline HIP 476 & 0.08 & 0.13 & -0.25 & 0.33 & 0.04 & $\ldots$ & 0.18 & 0.02 & $\ldots$ & RGB \\
\hline HIP 4197 & 0.19 & 0.09 & -0.68 & 0.48 & 0.01 & 0.17 & 0.04 & 0.06 & 22 & $\mathrm{RC} / \mathrm{RGB}$ \\
\hline HIP 8404 & 0.11 & 0.11 & -0.45 & 0.05 & -0.16 & -0.22 & 0.06 & -0.07 & 15 & RC \\
\hline HIP 13339 & -0.04 & 0.08 & -0.39 & 0.03 & -0.23 & -0.30 & 0.13 & -0.04 & 12 & RHB \\
\hline HIP 19611 & 0.04 & 0.12 & -0.31 & 0.03 & -0.13 & -0.05 & 0.14 & 0.05 & 30 & RGB/RHB \\
\hline HIP 19740 & 0.02 & 0.12 & -0.47 & 0.41 & -0.17 & -0.28 & 0.16 & -0.01 & 14 & RHB \\
\hline HIP 27280 & 0.02 & 0.10 & -0.38 & 0.26 & -0.12 & -0.14 & 0.15 & 0.00 & 30 & RGB/RHB \\
\hline HIP 38801 & 0.04 & 0.13 & -0.37 & 0.63 & 0.11 & 0.22 & 0.29 & 0.24 & $\ldots$ & HB \\
\hline HIP 39326 & 0.10 & 0.21 & -0.18 & 0.43 & 0.23 & 0.36 & 0.01 & 0.09 & $\ldots$ & SG \\
\hline HIP 44154 & 0.08 & 0.08 & -0.45 & 0.19 & -0.20 & -0.20 & 0.14 & 0.04 & 16 & RHB \\
\hline HIP 45033 & -0.01 & 0.10 & -0.44 & 0.33 & -0.18 & -0.14 & 0.13 & -0.01 & 10 & RHB \\
\hline HIP 45158 & 0.14 & 0.07 & -0.35 & 0.36 & 0.16 & 0.09 & 0.26 & 0.25 & 9 & $\mathrm{RC}$ \\
\hline HIP 51179 & 0.09 & 0.11 & -0.47 & 0.46 & -0.08 & 0.05 & 0.13 & 0.00 & 30 & RHB \\
\hline HIP 51487 & 0.06 & 0.15 & -0.04 & 0.14 & $\ldots$ & 0.23 & 0.29 & -0.06 & $\ldots$ & MS \\
\hline HIP 54048 & 0.04 & 0.11 & -0.59 & 0.37 & -0.11 & -0.19 & 0.15 & 0.05 & 10 & RHB \\
\hline HIP 56194 & 0.10 & 0.04 & -0.48 & 0.35 & -0.12 & -0.15 & 0.06 & 0.01 & 20 & RC/RGB \\
\hline HIP 57748 & 0.10 & 0.02 & -0.20 & 0.09 & 0.02 & 0.11 & -0.30 & -0.11 & 6 & RHB \\
\hline HIP 60140 & 0.05 & 0.02 & -0.49 & 0.18 & -0.25 & -0.10 & 0.16 & 0.11 & 20 & $\mathrm{RHB} / \mathrm{RC}$ \\
\hline HIP 60485 & 0.06 & 0.08 & -0.47 & 0.52 & -0.08 & -0.06 & 0.20 & 0.00 & 25 & - \\
\hline HIP 65900 & 0.03 & 0.08 & -0.03 & $\ldots$ & $\ldots$ & 0.01 & 0.07 & 0.19 & $\ldots$ & MS \\
\hline HIP 78990 & 0.02 & 0.16 & -0.42 & 0.50 & -0.03 & -0.24 & 0.16 & -0.06 & 15 & RHB \\
\hline HIP 80309 & 0.14 & 0.11 & -0.33 & 0.47 & -0.03 & -0.12 & 0.03 & 0.08 & 20 & RGB \\
\hline HIP 80543 & 0.10 & 0.09 & 0.01 & 0.04 & $\ldots$ & 0.09 & -0.05 & -0.07 & $\ldots$ & SG \\
\hline HIP 85715 & 0.12 & 0.03 & -0.42 & 0.41 & -0.03 & -0.05 & 0.08 & -0.06 & 25 & $\mathrm{RC} / \mathrm{RHB}$ \\
\hline HIP 89008 & 0.05 & 0.06 & -0.36 & 0.04 & -0.31 & -0.28 & 0.00 & -0.17 & 25 & RGB/RHB \\
\hline HIP 89095 & 0.10 & 0.14 & -0.40 & 0.40 & -0.19 & -0.15 & -0.11 & -0.06 & $\ldots$ & HB? \\
\hline HIP 90906 & 0.14 & 0.09 & -0.59 & 0.51 & -0.21 & -0.06 & -0.10 & -0.12 & 20 & RC/RHB \\
\hline HIP 91985 & 0.05 & 0.12 & -0.41 & 0.62 & -0.19 & -0.15 & 0.06 & -0.01 & 30 & HB? \\
\hline HIP 92827 & 0.13 & 0.00 & -0.75 & 0.72 & -0.24 & -0.10 & -0.12 & -0.09 & 22 & $\mathrm{RC}$ \\
\hline HIP 93940 & 0.12 & 0.09 & -0.10 & $\ldots$ & -0.02 & 0.11 & -0.14 & 0.07 & $\ldots$ & SG \\
\hline HIP 94598 & 0.10 & 0.09 & -0.63 & 0.49 & -0.13 & -0.15 & 0.11 & 0.00 & 17 & RHB/eAGB \\
\hline HIP 94779 & 0.09 & 0.04 & -0.53 & 0.14 & -0.28 & -0.15 & -0.10 & -0.06 & 15 & RC/RHB \\
\hline HIP 100274 & 0.02 & 0.05 & -0.42 & 0.24 & -0.05 & -0.09 & 0.06 & 0.02 & 20 & $\mathrm{RC} / \mathrm{RGB}$ \\
\hline HIP 103004 & 0.06 & 0.10 & -0.26 & 0.50 & -0.10 & 0.11 & 0.12 & 0.13 & 20 & RHB \\
\hline HIP 103734 & 0.05 & -0.01 & -0.34 & 0.28 & -0.16 & -0.23 & -0.06 & -0.12 & 30 & RGB \\
\hline HIP 113610 & 0.08 & 0.13 & -0.56 & 0.45 & -0.08 & -0.16 & 0.13 & 0.02 & 20 & RHB \\
\hline HIP 114809 & 0.13 & 0.14 & -0.27 & 0.32 & 0.11 & 0.16 & 0.05 & 0.11 & $\ldots$ & RHB \\
\hline HIP 118209 & 0.03 & 0.06 & -0.42 & 0.25 & -0.15 & -0.29 & 0.17 & 0.08 & 22 & RGB/RHB \\
\hline HD 9097 & 0.05 & -0.04 & -0.06 & 0.11 & $\ldots$ & 0.17 & 0.01 & 0.25 & $\ldots$ & MS/SG \\
\hline HD 84686 & 0.13 & -0.09 & -0.10 & 0.15 & 0.14 & 0.05 & -0.26 & 0.24 & 10 & RHB \\
\hline HD 96780 & 0.12 & 0.14 & -0.04 & & 0.18 & 0.15 & 0.04 & 0.08 & $\ldots$ & $\mathrm{MS} / \mathrm{SG}$ \\
\hline HD 101014 & 0.04 & -0.02 & -0.13 & 0.20 & $\ldots$ & 0.04 & 0.00 & -0.06 & $\ldots$ & $\mathrm{MS} / \mathrm{SG}$ \\
\hline HD 141770 & 0.03 & 0.00 & -0.04 & 0.20 & 0.03 & 0.15 & 0.03 & 0.06 & $\ldots$ & $\mathrm{MS} / \mathrm{SG}$ \\
\hline
\end{tabular}


Table 7

(Continued)

\begin{tabular}{|c|c|c|c|c|c|c|c|c|c|c|}
\hline Stars & {$[\mathrm{Si} \mathrm{I} / \mathrm{Fe}]$} & {$[\mathrm{Ca} / \mathrm{Fe}]$} & {$[\mathrm{C} / \mathrm{Fe}]_{\mathrm{CH}}$} & {$[\mathrm{N} / \mathrm{Fe}]_{\mathrm{CN}}$} & {$[\mathrm{O} \mathrm{I} / \mathrm{Fe}]$} & {$[\mathrm{O} \mathrm{I} / \mathrm{Fe}]_{\mathrm{NLTE}}$} & {$[\mathrm{La} \mathrm{II} / \mathrm{Fe}]$} & {$[\mathrm{Eu}$ II $/ \mathrm{Fe}]$} & ${ }^{12} \mathrm{C} /{ }^{13} \mathrm{C}$ & Evol. Phase \\
\hline HD 166310 & 0.04 & 0.02 & -0.39 & 0.14 & $\ldots$ & 0.15 & 0.02 & 0.24 & $\ldots$ & MS \\
\hline HD 221744 & -0.01 & 0.13 & -0.15 & 0.35 & 0.14 & 0.04 & 0.12 & 0.14 & $\ldots$ & MS \\
\hline HD 235802 & 0.12 & 0.02 & -0.26 & 0.22 & $\ldots$ & 0.20 & $\ldots$ & -0.17 & $\ldots$ & MS \\
\hline HD 242647 & 0.02 & 0.11 & -0.17 & 0.11 & $\ldots$ & 0.33 & 0.17 & 0.20 & $\ldots$ & MS \\
\hline HD 243170 & 0.03 & 0.09 & -0.20 & $\ldots$ & $\ldots$ & 0.03 & $\ldots$ & -0.03 & $\ldots$ & SG \\
\hline BD-14 1413 & 0.19 & 0.19 & -0.17 & $\ldots$ & $\ldots$ & 0.35 & 0.00 & 0.14 & $\ldots$ & SG \\
\hline BD+31 2565 & 0.07 & 0.03 & -0.08 & $\ldots$ & $\ldots$ & 0.10 & 0.25 & 0.04 & $\ldots$ & MS \\
\hline $\mathrm{BD}+322190$ & 0.03 & 0.00 & -0.01 & 0.23 & $\ldots$ & 0.05 & -0.13 & 0.07 & $\ldots$ & $\mathrm{MS} / \mathrm{SG}$ \\
\hline $\mathrm{BD}+412221$ & 0.05 & 0.02 & 0.04 & 0.19 & 0.13 & 0.04 & -0.05 & 0.06 & $\ldots$ & $\mathrm{MS} / \mathrm{SG}$ \\
\hline $\mathrm{BD}+451958$ & 0.02 & -0.02 & -0.24 & 0.29 & $\ldots$ & 0.04 & -0.14 & 0.02 & $\ldots$ & MS \\
\hline $\mathrm{BD}+452032$ & 0.08 & 0.12 & -0.29 & 0.14 & -0.02 & 0.20 & 0.14 & 0.38 & $\ldots$ & SG \\
\hline $\mathrm{BD}+542710$ & 0.16 & 0.11 & -0.60 & 0.52 & -0.22 & -0.05 & 0.10 & -0.01 & 20 & $\mathrm{RC}$ \\
\hline TYC 3720-324-1 & 0.10 & -0.05 & -0.05 & 0.16 & $\ldots$ & 0.04 & 0.07 & -0.29 & $\ldots$ & $\mathrm{MS} / \mathrm{SG}$ \\
\hline
\end{tabular}

Note. Due to lack of parallax information we were unable to investigate the kinematical parameters of BD+27 2057 but by taking into account the previous studies, e.g., Stetson \& Aikman (1987), Upgren (1962, 1963), and Tautvaišienè et al. (2001), we list it as a thick-disk member in the table.

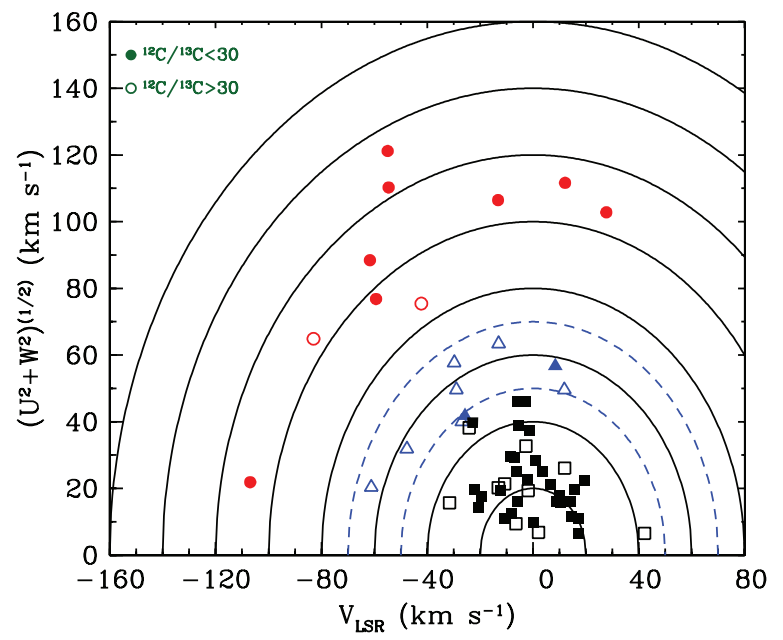

Figure 13. Toomre diagram of the program stars. Solid lines indicate $V_{\text {tot }}$ in steps of $20 \mathrm{~km} \mathrm{~s}^{-1}$. The dashed lines at 50 and $70 \mathrm{~km} \mathrm{~s}^{-1}$ denote the approximate thin-thick-disk separation. Thin-, thin-/thick-, and thick-disk stars are represented by (black) squares, (blue) triangles, and (red) circles. Filled and open symbols represent whether ${ }^{12} \mathrm{C} /{ }^{13} \mathrm{C}$ is detected in the spectrum of the related star (representative filled and open symbols and their meaning are also shown in the figure).

(A color version of this figure is available in the online journal.)

Leeuwen (2007), who recently published new reduction results of Hipparcos data. We either adopted the RVs already available in the literature or we measured new values as described in Section 2.2. We used the matrix equations given by Johnson \& Soderblom (1987) and calculated the Galactic velocity components $U_{\mathrm{LSR}}$ (positive toward the Galactic center, $V_{\mathrm{LSR}}$ (positive in the direction of the Galactic rotation), $W_{\mathrm{LSR}}$ (positive toward the NGP), and their uncertainties $\left(\sigma_{U_{\mathrm{LSR}}}, \sigma_{V_{\mathrm{LSR}}}, \sigma_{W_{\mathrm{LSR}}}\right)$ with respect to the local standard of rest (LSR). Correction for the solar motion was made by adopting the values $(U, V, W)_{\odot}=(+10.00$, $+5.25,+7.17)$ from Dehnen \& Binney (1998). The results are given in Table 8.

In Figure 13 we show these kinematics in a Toomre diagram. This kind of plot gives the kinematical distribution of the stars in terms of their combined vertical and radial kinetic energies as a function of their rotational energies. The solid curves of Figure 13 are lines of constant total space velocity, $V_{\text {tot }}=\left(U_{\text {LSR }}^{2}+V_{\text {LSR }}^{2}+W^{2}{ }_{\text {LSR }}\right)^{1 / 2}$. The dashed line represents the $V_{\mathrm{tot}}=70 \mathrm{~km} \mathrm{~s}^{-1}$, which approximately separates the thin- and thick-disk populations of the Galaxy (e.g., Bensby \& Feltzing 2010; Nissen 2004). Stars with total space velocities of $70 \mathrm{~km} \mathrm{~s}^{-1}<V_{\text {tot }}<180 \mathrm{~km} \mathrm{~s}^{-1}$ are considered to be probable thick disks, those in the range $50 \mathrm{~km} \mathrm{~s}^{-1}<V_{\text {tot }}<70 \mathrm{~km} \mathrm{~s}^{-1}$ are called transition (thin/thick) objects, and those with $V_{\text {tot }}<$ $50 \mathrm{~km} \mathrm{~s}^{-1}$ are probable thin-disk members of the Galaxy.

Inspection of Figure 13 reveals that our sample is dominated by thin-disk stars. Out of the 58 stars with kinematic information, 39 (the squares) probably reside in the thin disk, 10 probably are in the thick disk, and 9 are kinematic transition objects whose thin/thick status is ambiguous. In Figure 13 we also use filled and open version of the symbols to indicate the detection status of ${ }^{13} \mathrm{CN}$ features in individual stars: those with ${ }^{12} \mathrm{C} /{ }^{13} \mathrm{C}<30$, and those with no detection of ${ }^{13} \mathrm{CN}$ (thus ${ }^{12} \mathrm{C} /{ }^{13} \mathrm{C}>30$ ), respectively. We will consider the carbon isotopic ratio distribution further in Section 7.

Looking at the kinematics of our stars in more detail, in Figure 14 we show the correlations between total space velocity $V_{\text {tot }}$ and eccentricity, and between the velocity component of $W_{\text {LSR }}$ and eccentricity. The eccentricities were calculated using the method described in Dinescu et al. (1999). The solar circle radius of $R_{0}=8.0 \mathrm{kpc}$ around the Galactic center and an LSR rotation velocity of $\Theta=220 \mathrm{~km} \mathrm{~s}^{-1}$ were adopted. As expected, the total velocity is strongly correlated with eccentricity in our sample. However, the relationship between the vertical velocity and eccentricity is not obvious beyond increased scatter in $V_{\text {tot }}$ at large eccentricities. Several of our thick-disk stars have low $\left|W_{\mathrm{LSR}}\right|$ velocities $\left(\lesssim 20 \mathrm{~km} \mathrm{~s}^{-1}\right)$. Given that $W_{\mathrm{LSR}}$ is proportional to $z_{\max }$, the maximum vertical distance of a star to the Galactic plane, these particular stars with $W_{\mathrm{LSR}}$ apparently reside close to the Galactic plane.

The space motions and derived metallicities of our program stars are related. In Figure 15, we plot the three space velocity components against $[\mathrm{Fe} / \mathrm{H}]$ in panels (a), (b), and (c). The larger dispersions of the $U_{\mathrm{LSR}}, V_{\mathrm{LSR}}$, and $W_{\mathrm{LSR}}$ velocities in the true thick-disk stars is clear from inspection of these panels. Useful velocity limits in order to separate the thinand thick-disk stars appear to be $\left|W_{\mathrm{LSR}}\right| \approx+20 \mathrm{~km} \mathrm{~s}^{-1}$ and $V_{\mathrm{LSR}}<-40 \mathrm{~km} \mathrm{~s}^{-1}$. However, all three velocity components that comprise total space velocity must be taken into account in assessing the Galactic membership of individual stars. In panel (d) of Figure 15 we plot $[\mathrm{Fe} / \mathrm{H}]$ versus eccentricity, and it is clear that lower metallicity stars in our sample generally have 
Table 8

Kinematics

\begin{tabular}{|c|c|c|c|c|c|c|c|}
\hline Stars & $\begin{array}{c}U_{\mathrm{LSR}} \\
\left(\mathrm{km} \mathrm{s}^{-1}\right)\end{array}$ & $\begin{array}{c}\sigma_{U_{\mathrm{LSR}}} \\
\left(\mathrm{km} \mathrm{s}^{-1}\right)\end{array}$ & $\begin{array}{c}V_{\mathrm{LSR}} \\
\left(\mathrm{km} \mathrm{s}^{-1}\right)\end{array}$ & $\begin{array}{c}\sigma_{V_{\mathrm{LSR}}} \\
\left(\mathrm{km} \mathrm{s}^{-1}\right)\end{array}$ & $\begin{array}{c}W_{\mathrm{LSR}} \\
\left(\mathrm{km} \mathrm{s}^{-1}\right)\end{array}$ & $\begin{array}{c}\sigma_{W_{\mathrm{LSR}}} \\
\left(\mathrm{km} \mathrm{s}^{-1}\right)\end{array}$ & Membership \\
\hline HIP 62325 & 117.5 & 1.6 & -55.0 & 0.7 & 29.6 & 0.5 & $\mathrm{Tk}$ \\
\hline HIP 3031 & 110.1 & 0.7 & -54.6 & 0.7 & 6.9 & 0.7 & $\mathrm{Tk}$ \\
\hline HIP 5104 & -108.0 & 29.2 & 12.1 & 2.6 & 28.3 & 11.3 & $\mathrm{Tk}$ \\
\hline HIP 71837 & -21.1 & 1.0 & -106.9 & 4.5 & 5.8 & 1.1 & $\mathrm{Tk}$ \\
\hline HIP 66892 & -88.2 & 11.0 & -61.7 & 6.5 & -6.9 & 2.8 & $\mathrm{Tk}$ \\
\hline HIP 72631 & 99.2 & 1.1 & -13.2 & 0.4 & 38.7 & 1.1 & $\mathrm{Tk}$ \\
\hline HIP 45412 & -100.2 & 5.2 & 27.7 & 2.0 & -22.9 & 5.0 & Tk \\
\hline HIP 4960 & 46.7 & 5.2 & -83.0 & 10.4 & 45.0 & 9.2 & Tk \\
\hline HIP 115839 & -49.8 & 2.5 & -59.4 & 2.0 & 58.5 & 2.2 & Tk \\
\hline HIP 58269 & 74.1 & 7.2 & -42.2 & 5.2 & 14.5 & 11.3 & Tk \\
\hline HIP 82014 & -57.6 & 7.0 & -29.8 & 2.0 & -4.4 & 0.5 & $\mathrm{Tk} / \mathrm{Tn}$ \\
\hline HIP 11924 & 63.3 & 2.1 & -13.0 & 1.1 & -3.2 & 2.3 & $\mathrm{Tk} / \mathrm{Tn}$ \\
\hline HIP 46325 & 13.2 & 2.1 & -61.1 & 5.4 & 15.5 & 1.5 & $\mathrm{Tk} / \mathrm{Tn}$ \\
\hline HIP 70344 & 49.6 & 2.4 & -29.0 & 2.9 & 1.0 & 6.1 & $\mathrm{Tk} / \mathrm{Tn}$ \\
\hline HIP 98587 & 51.8 & 8.6 & 8.4 & 4.0 & -23.1 & 7.8 & $\mathrm{Tk} / \mathrm{Tn}$ \\
\hline HIP 70341 & -24.7 & 1.9 & -47.7 & 2.7 & -20.1 & 1.3 & $\mathrm{Tk} / \mathrm{Tn}$ \\
\hline HIP 57535 & 49.4 & 5.1 & 11.9 & 1.4 & -4.6 & 1.5 & $\mathrm{Tk} / \mathrm{Tn}$ \\
\hline HIP 75823 & -41.6 & 17.0 & -25.8 & 8.5 & 3.9 & 13.2 & $\mathrm{Tk} / \mathrm{Tn}$ \\
\hline HIP 60873 & -27.4 & 9.3 & -26.9 & 4.9 & -29.3 & 1.2 & $\mathrm{Tk} / \mathrm{Tn}$ \\
\hline HIP 57748 & 46.1 & 4.8 & -5.9 & 1.4 & -1.9 & 1.9 & Tn \\
\hline HIP 27280 & 43.9 & 1.9 & -3.0 & 1.0 & -14.0 & 1.3 & Tn \\
\hline HIP 4197 & 31.2 & 4.0 & -22.8 & 5.1 & -24.6 & 9.4 & Tn \\
\hline HIP 93940 & -35.2 & 1.2 & -24.1 & 1.2 & -14.7 & 2.0 & $\mathrm{Tn}$ \\
\hline HIP 114809 & 1.7 & 0.6 & 42.2 & 1.7 & -6.3 & 1.2 & $\operatorname{Tn}$ \\
\hline HIP 118209 & 38.9 & 0.7 & -5.5 & 0.5 & 1.7 & 0.8 & $\operatorname{Tn}$ \\
\hline HIP 103004 & 35.4 & 1.2 & -1.4 & 1.9 & 12.7 & 0.5 & $\operatorname{Tn}$ \\
\hline HIP 65900 & -12.6 & 1.3 & -31.6 & 1.6 & -9.4 & 0.5 & $\operatorname{Tn}$ \\
\hline HIP 89095 & -31.4 & 5.0 & -2.7 & 5.1 & -9.3 & 2.2 & $\operatorname{Tn}$ \\
\hline HIP 113610 & -29.0 & 4.6 & -8.3 & 0.9 & 6.7 & 1.6 & Tn \\
\hline HIP 92827 & -27.7 & 3.6 & -7.2 & 4.6 & -9.2 & 1.7 & $\operatorname{Tn}$ \\
\hline HIP 51179 & -2.3 & 1.6 & 19.5 & 0.4 & -22.4 & 0.7 & $\operatorname{Tn}$ \\
\hline HIP 80309 & -19.7 & 1.6 & -22.0 & 1.6 & 0.4 & 1.4 & $\operatorname{Tn}$ \\
\hline HIP 39326 & 25.7 & 0.4 & 11.9 & 0.4 & 4.2 & 0.4 & Tn \\
\hline HIP 13339 & 26.8 & 1.6 & 1.0 & 1.2 & -9.0 & 1.0 & $\mathrm{Tn}$ \\
\hline HIP 94779 & -17.2 & 0.2 & -19.4 & 0.9 & -3.7 & 0.3 & $\mathrm{Tn}$ \\
\hline HIP 90906 & -25.0 & 4.0 & -6.4 & 3.1 & 0.9 & 1.1 & $\mathrm{Tn}$ \\
\hline HIP 78990 & 15.5 & 0.9 & 3.7 & 0.3 & -19.7 & 0.8 & $\operatorname{Tn}$ \\
\hline HIP 45158 & -19.7 & 1.3 & 15.8 & 1.8 & 0.7 & 0.7 & $\operatorname{Tn}$ \\
\hline HIP 56194 & -5.2 & 1.3 & -20.6 & 2.1 & 13.2 & 1.8 & $\operatorname{Tn}$ \\
\hline HD 96780 & -20.1 & $\ldots$ & -13.1 & $\ldots$ & 1.9 & $\ldots$ & $\operatorname{Tn}$ \\
\hline HIP 80543 & -13.4 & 3.3 & -10.7 & 2.1 & -16.7 & 3.2 & Tn \\
\hline HIP 44154 & -18.5 & 1.5 & -12.4 & 0.5 & 5.6 & 1.4 & $\operatorname{Tn}$ \\
\hline HIP 19740 & 22.5 & 1.0 & -2.3 & 0.8 & -0.8 & 1.1 & $\operatorname{Tn}$ \\
\hline HIP 94598 & 21.3 & 1.4 & 6.8 & 1.7 & -0.1 & 0.9 & $\mathrm{Tn}$ \\
\hline HIP 100274 & 12.2 & 1.2 & 14.3 & 1.7 & 10.5 & 0.8 & $\operatorname{Tn}$ \\
\hline HIP 60485 & 17.1 & 0.4 & 10.2 & 0.4 & -5.6 & 0.8 & $\operatorname{Tn}$ \\
\hline HIP 91985 & 10.6 & 0.7 & 17.4 & 1.9 & -3.3 & 1.6 & $\mathrm{Tn}$ \\
\hline HIP 51487 & 18.3 & 0.4 & -1.8 & 0.7 & -6.3 & 0.5 & Tn \\
\hline HIP 45033 & 15.2 & 1.4 & 10.3 & 0.7 & 4.4 & 1.3 & $\operatorname{Tn}$ \\
\hline HIP 8404 & 7.3 & 1.0 & 14.7 & 0.8 & 8.8 & 1.7 & $\operatorname{Tn}$ \\
\hline HIP 19611 & 4.8 & 0.5 & 17.4 & 1.6 & 4.2 & 0.7 & $\mathrm{Tn}$ \\
\hline HIP 54048 & 15.9 & 0.7 & 8.8 & 0.4 & 2.2 & 0.4 & Tn \\
\hline HIP 103734 & 14.5 & 1.0 & -5.9 & 4.9 & 6.9 & 0.7 & Tn \\
\hline HIP 89008 & -7.3 & 0.9 & -10.7 & 1.7 & -8.2 & 0.9 & Tn \\
\hline HIP 85715 & -11.1 & 1.1 & -8.1 & 1.4 & -5.8 & 1.0 & $\mathrm{Tn}$ \\
\hline HIP 476 & -9.4 & 0.8 & -6.4 & 1.4 & -0.4 & 1.5 & $\mathrm{Tn}$ \\
\hline HIP 60140 & -8.7 & 4.1 & 0.2 & 1.7 & 4.2 & 0.9 & $\operatorname{Tn}$ \\
\hline HIP 38801 & -2.5 & 4.8 & 2.1 & 4.5 & 6.3 & 5.6 & $\operatorname{Tn}$ \\
\hline
\end{tabular}

Note. "Tk," "Tn," and "Tk/Tn" stand for thick-, thin-, and thick-/thin-disk stars, respectively.

more eccentric orbits. This is similar to the relation recently suggested by Lee et al. (2011). However, their study considered only thick-disk stars with sub-solar metallicities $([\mathrm{Fe} / \mathrm{H}]<$ $-0.3)$. We stress here that stars with thick-disk kinematics (including eccentricity) but $[\mathrm{Fe} / \mathrm{H}]>-0.3$ have no obvious metallicity-kinematics connections.

In Figure 16 we explore the relationship between our $\alpha$-element abundances $([\alpha / \mathrm{Fe}]=([\mathrm{Ca} / \mathrm{Fe}]+[\mathrm{Si} / \mathrm{Fe}]) / 2)$, 

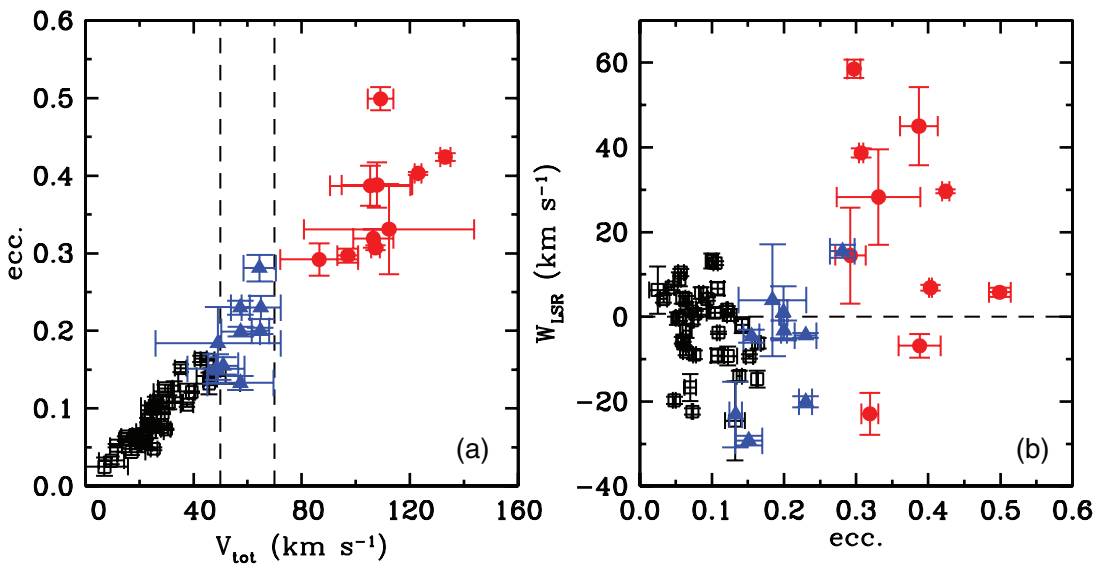

Figure 14. (a) Correlation of Galactic orbital eccentricity ecc. with total space velocity $V_{\text {tot }}$. (b) Correlation of vertical velocity component $W_{\mathrm{LSR}}$ with eccentricity. The symbol colors are the same as those of Figure 13.

(A color version of this figure is available in the online journal.)
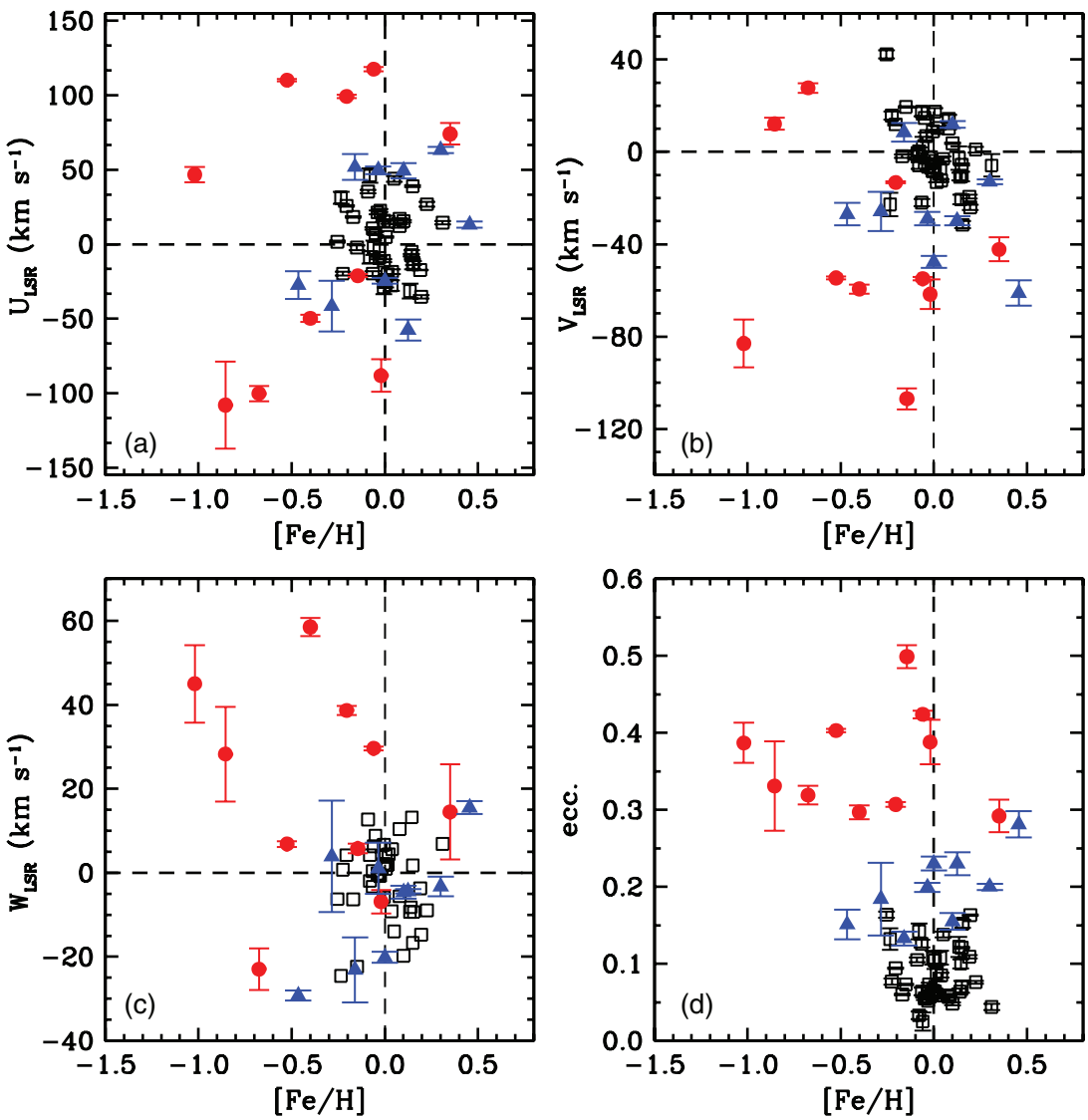

Figure 15. Correlations of kinematic components with $[\mathrm{Fe} / \mathrm{H}]$ metallicity: $U_{\mathrm{LSR}}$ in panel (a); $V_{\mathrm{LSR}}$ in panel (b); $W_{\mathrm{LSR}}$ in panel (c); and eccentricity in panel (d). The symbol colors are the same as those of Figure 13.

(A color version of this figure is available in the online journal.)

metallicity, and kinematics. In panel (a) we correlate $[\mathrm{Fe} / \mathrm{H}]$ with $[\alpha / \mathrm{Fe}]$. The well-documented rise in relative $\alpha$-element abundances with decreasing metallicity in disk populations is reproduced in our relatively small sample. All of our thick-disk stars with $[\mathrm{Fe} / \mathrm{H}]<-0.3$ have $[\alpha / \mathrm{Fe}] \gtrsim+0.2$. Although our sample is small, there seems to be a clear separation between the thick- and thin-disk stars for the metallicities of $[\mathrm{Fe} / \mathrm{H}]<-0.3$ as indicated in larger surveys (e.g., Reddy et al. 2006). Stars with $[\mathrm{Fe} / \mathrm{H}]>-0.3$ show similar $[\alpha / \mathrm{Fe}]$ ratios regardless of their membership either in the thick or the thin disk. In panel (b) we plot the $[\alpha / \mathrm{Fe}]$ versus metallicity. This relationship can be compared with the $W_{\text {LSR }}$ versus $[\mathrm{Fe} / \mathrm{H}]$ plot shown in panel (d) of Figure 15. They tell essentially the same story, linking the kinematics, metallicities, and $\alpha$-element abundances of our sample.

\section{EVOLUTIONARY STATUS}

We estimated the probable evolutionary stages of our program stars by taking into account their loci in the $T_{\text {eff }}-\log g$ plane, their absolute magnitudes (luminosities), and their ${ }^{12} \mathrm{C} /{ }^{13} \mathrm{C}$ values derived from our spectral analysis. In Figure 17 we show the 

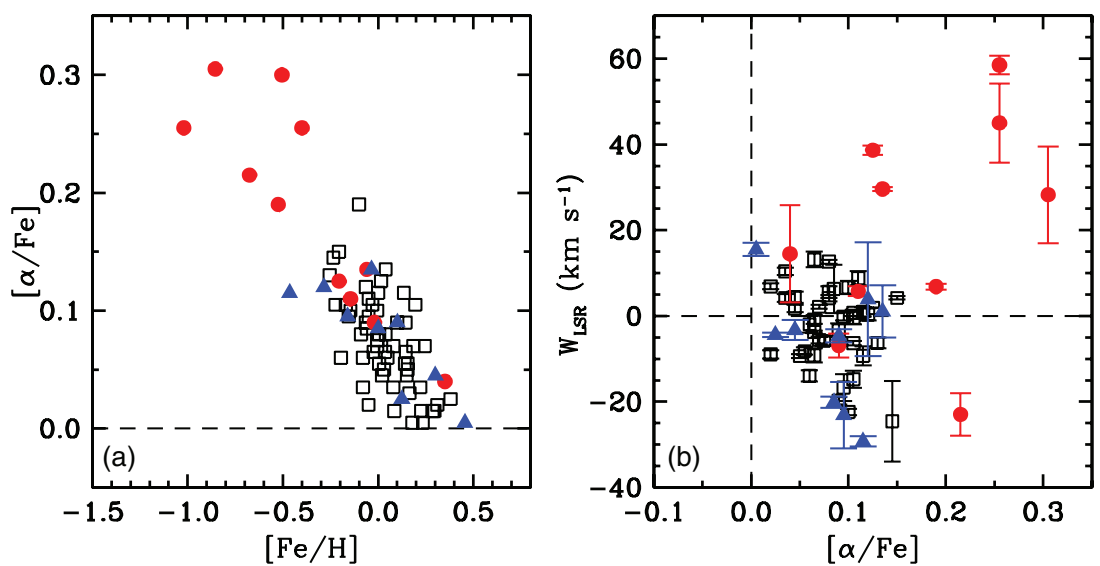

Figure 16. (a) Correlation of relative $[\alpha / \mathrm{Fe}]$ abundance ratios with respect to metallicity $[\mathrm{Fe} / \mathrm{H}]$. (b) Comparison of vertical velocity component $W_{\mathrm{LSR}}$ with $[\alpha / \mathrm{Fe}]$ values. The symbol colors are the same as those of Figure 13.

(A color version of this figure is available in the online journal.)

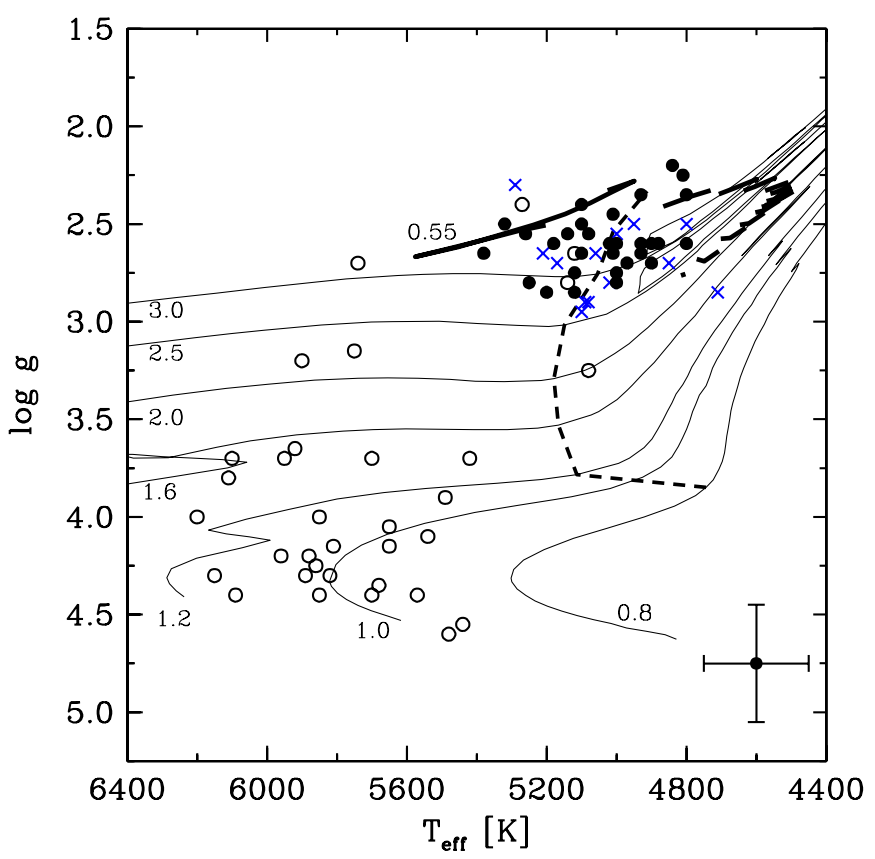

Figure 17. Evolutionary states of our program stars are shown on the spectroscopic $\log g-T_{\text {eff }}$ diagram. Stars with ${ }^{12} \mathrm{C} /{ }^{13} \mathrm{C} \leqslant 20,20<{ }^{12} \mathrm{C} /{ }^{13} \mathrm{C} \leqslant 30$, and ${ }^{12} \mathrm{C} /{ }^{13} \mathrm{C}>30$ (no detection) are shown by filled circles, (blue) crosses, and open circles, respectively.

(A color version of this figure is available in the online journal.)

$T_{\text {eff }}-\log g$ diagram, using different symbols to denote stars with and without detected ${ }^{13} \mathrm{CN}$ spectral features. Although we selected our targets as luminosity class-III stars, our atmospheric analyses revealed about $1 / 3$ of our sample to be either MS or SGB stars. These are evident in the figure as higher temperature, higher gravity stars with weak/absent ${ }^{13} \mathrm{CN}$.

In Figure 17 we have also shown theoretical evolutionary tracks from Bertelli et al. (2008) $(Y=0.26, Z=0.02$; note that we do have a few low-metallicity stars in our sample but for simplicity we plot only tracks with a single metallicity). The evolutionary-track masses displayed here are from 0.8 to $3 M_{\odot}$. For tracks with $0.8-2 M_{\odot}$ the evolutionary phases go up to the RGB tip (at lower temperatures than displayed here), while the 2.5 and $3 M_{\odot}$ tracks proceed up to the first thermal pulse stage. The base of the RGB, including masses up to $4 M_{\odot}$, is also shown with a dashed line, which is constructed from the data of Bertelli et al. (2008). We have also included HB evolutionary tracks for the mass range $0.55-2 M_{\odot}$ (denoted by the thick black lines). Only $0.55 M_{\odot}$ HB track evolves up to the early asymptotic giant branch (AGB) phase. Other HB tracks start from the ZAHB and go up to the maximum He-burning phase. HB tracks with masses higher than $0.55 M_{\odot}$ make an arc shape in Figure 17. This arc covers a temperature range $4500 \mathrm{~K}$ $\lesssim T_{\text {eff }} \lesssim 4900 \mathrm{~K}$, and thus essentially defines the $\mathrm{RC}$ region. $\mathrm{RC}$ stars are the reddest $\mathrm{HB}$ stars with higher masses than normal RHB stars. Both of them are at the core He-burning stage and all belong to the RHB class in general.

In Section 3.1 we compared spectroscopic surface gravities with theoretical ones (Figure 4), suggesting that, for most of our targets, the masses are $\sim 2 M_{\odot}$. This is consistent with the inferred evolutionary-track masses of the warmer $\left(T_{\text {eff }}>\right.$ $5300 \mathrm{~K})$, higher gravity $(\log g>3.0)$ stars in Figure 17. The SG stars should not yet have convectively mixed envelopes, so it is not surprising that these objects also do not have detectable ${ }^{13} \mathrm{CN}$ lines and hence ${ }^{12} \mathrm{C} /{ }^{13} \mathrm{C}>30$ (e.g., Thorén et al. 2004).

For almost all of the stars with $T_{\text {eff }}<5300 \mathrm{~K}$, the masses inferred from the evolutionary tracks in Figure 17 are $\gtrsim 3 M_{\odot}$. These implied masses are much larger than those of the warmer MS/SG program stars, and also are inconsistent with our earlier (Section 3.1) assertion that almost all of our stars have actual masses $\lesssim 2 M_{\odot}$. The implication is that our lower temperature stars are not SG stars on their first ascent up the RGB, but instead are post-RGB stars in the helium-burning RHB evolutionary stage. It is possible for RHBs to be confused with high-mass SGs, which can have similar absolute magnitudes to the RHBs. But such SG stars should be rare because the timescales for passage of $\gtrsim 3 M_{\odot}$ stars through the 4800-5300 K temperature domain $\left(\sim 10^{5}-10^{6}\right.$ years $)$ are short compared to the RHB Heburning timescales $\left(\sim 10^{8}\right.$ years) of less massive (thus probably more plentiful) stars. Our cooler program stars in general ought to be true members of the field RHB population.

For each program star we have estimated a probable evolutionary stage, taking into account its position in the $T_{\text {eff }}-\log g$ plane from arguments presented above, its absolute magnitude when parallax data are available, and its ${ }^{12} \mathrm{C} /{ }^{13} \mathrm{C}$ ratio. These estimates are given in the last column of Table 7 . We suggest that there are 18 RHB stars in our sample (5 thick disk and 13 thin disk), along with several RC and RC/RHB stars, most of them members of the thin disk. 


\section{SUMMARY AND DISCUSSION}

In this study we have determined atmospheric parameters and chemical compositions of field RHB candidates selected simply from their colors and absolute magnitudes. The original goal was to increase the sample size of bona fide RHB stars, and to try to understand the physical processes involved in the existence of these relatively rare highly evolved objects. We deliberately avoided kinematic biases by employing only photometric and spectral type information in selecting stars for observation.

We gathered high-resolution, high $\mathrm{S} / \mathrm{N}$ spectra of 129 candidate field RHB stars. Preliminary analyses eliminated 53 candidates from further consideration because they proved to be anomalously broad-lined rapidly rotating stars, or double-lined spectroscopic binaries, or stars for which we could not deduce reliable atmospheric parameters. For the remaining 76 program stars, we first determined values of $T_{\mathrm{eff}}, \log g, \xi_{\mathrm{t}}$, and $[\mathrm{Fe} / \mathrm{H}]$. Then we derived abundances for $\alpha$ elements $\mathrm{Si}$ and $\mathrm{Ca}$, neutroncapture elements $\mathrm{La}$ and $\mathrm{Eu}$, and proton-capture elements $\mathrm{Li}, \mathrm{C}$, $\mathrm{N}$, and $\mathrm{O}$. We also determined ${ }^{12} \mathrm{C} /{ }^{13} \mathrm{C}$ isotopic ratios, because detection of ${ }^{13} \mathrm{C}$ provides strong evidence of $\mathrm{CN}$-cycle $\mathrm{H}$-fusion and mixing associated with evolved stars. The derived fundamental stellar parameters $T_{\text {eff }}$ and $\log g$, along with the ${ }^{12} \mathrm{C} /{ }^{13} \mathrm{C}$ isotopic ratios, were used to estimate the evolutionary stages of our program stars. This evaluation suggests that about $20 \%$ of 76 program stars are true RHB members.

We then computed space velocity components for all of the program stars with available distances, proper motions, and RVs (either from the literature or from our own spectra). For each star we estimated Galactic population membership (thin disk, thick disk, and thin/thick transition) only from the kinematics. We examined correlations between kinematics and (1) overall metallicity, and (2) relative abundance ratios of the $\alpha$ elements (confirming these with abundance ratios of the neutron-capture elements). Even with our relatively small sample we recovered well-known differences between the thin- and thick-disk stars. Our thick-disk stars have higher space velocities and orbital eccentricities, lower metallicities, and larger $\alpha$-element ratios than do their thin-disk counterparts.

We did not take into account the kinematical constraints during the sample selection, and our moderately small survey of 76 RHB candidates turns out to contain only five probable thick-disk stars. These five true thick-disk RHBs are very similar to others already identified in the literature (e.g., Cottrell \& Sneden 1986; Tautvaišienè et al. 2001): they are mildly metalpoor, $\alpha$-enhanced (including high oxygen) stars that have low ${ }^{12} \mathrm{C} /{ }^{13} \mathrm{C}$ ratios. They also show little evidence for carbon depletion, as discussed in Section 4.3. Such stars have relatively low masses, and their appearance on the RHB (instead of the RC) is not surprising: mildly metal-poor GCs have well-populated RHBs.

A perhaps more interesting result is that our identified RHB sample is dominated by thin-disk, high-metallicity stars. These stars, with $T_{\text {eff }}>5000 \mathrm{~K}$, are too hot to be RC stars, which have temperatures lower than $4900 \mathrm{~K}$. They also have evolvedstar gravities: $\log g=2.2-2.8$, substantially smaller than the gravities of subgiants and MS stars (Figure 17). They show no obvious $\alpha$ and neutron-capture abundance anomalies compared to other thin-disk samples. Their proton-capture abundances are unremarkable compared to normal thin-disk RGB stars. In particular, they exhibit $[\mathrm{O} / \mathrm{Fe}] \sim 0$ and $[\mathrm{C} / \mathrm{Fe}] \sim-0.4$, and often low ${ }^{12} \mathrm{C} /{ }^{13} \mathrm{C}$ values, all consistent with expectations from past studies. But their residence in the RHB cannot be easily understood through standard stellar evolution considerations.

Both thick and the thin-disk RHBs have a range of ${ }^{12} \mathrm{C} /{ }^{13} \mathrm{C}$ ratios ranging from 5 to 30 (Table 7). Before discussing the ${ }^{12} \mathrm{C} /{ }^{13} \mathrm{C}$ ratios of our stars in detail, we review the physical processes that can affect ${ }^{12} \mathrm{C} /{ }^{13} \mathrm{C}$ ratios during stellar evolution in the next paragraphs.

As a low-mass star $\left(<2.25 M_{\odot}\right)$ evolves past the MS and SG evolutionary stages, the first dredge-up starts at the base of the RGB (e.g., Iben 1964, 1965, 1967b), accompanied by convective envelope expansion toward inner layers of the star. This will bring $\mathrm{CN}$-cycle processed material up to the outer layers, thus resulting in surface abundance alterations of the LiCNO group. Fragile $\mathrm{Li}$ is severely depleted first. As the convection extends into deeper inner regions, it passes through the transition region which separates the region of ${ }^{12} \mathrm{C}$ converted into ${ }^{14} \mathrm{~N}$. In standard dredge-up theories, mixing of the processed and unprocessed elements results in depletion of surface ${ }^{12} \mathrm{C}$, lowering the ${ }^{12} \mathrm{C} /{ }^{13} \mathrm{C}$ ratio to $\sim 20-30$ from its (assumed) initial solar value of 90 (Charbonnel 1994; Charbonnel et al. 1998; Gratton et al. 2000), while the surface ${ }^{14} \mathrm{~N}$ values increase. First dredgeup phenomenon leaves a mean molecular gradient ( $\mu$-barrier) behind which prevents further mixing (e.g., Charbonnel et al. 1998).

However, previous observational studies have shown that ${ }^{12} \mathrm{C} /{ }^{13} \mathrm{C}$ ratios as low as the $\mathrm{CN}$-cycle equilibrium value of 3.4 (e.g., Sneden et al. 1986; Cottrell \& Sneden 1986; Gratton et al. 2000; Tautvaišienè et al. 2001), which obviously requires a noncanonical mixing process during the RGB phase. Recent stellar evolution studies have attempted to solve this extra-mixing problem by offering physical mechanisms such as rotationinduced mixing (Zahn 1992), cool bottom processing (CBP; Boothroyd \& Sackmann 1999) and thermohaline instability plus rotation-induced mixing (Charbonnel \& Zahn 2007; Charbonnel \& Lagarde 2010). These ideas provide an extra-mixing process that sets in after the " $\mu$-barrier elimination" which occurs after the convective envelope base recedes toward the surface. Then, the H-burning shell eliminates the composition discontinuity left by the convective envelope and enables extra-mixing processes to come into play. For low-mass stars, this stage of evolution is also called the "RGB luminosity function bump (LFB)" (Gratton et al. 2000; Charbonnel \& Lagarde 2010) at which the H-burning shell burns the newly supplied fuel as it expands outward. This stage slows down the evolution and causes a temporary luminosity drop along the RGB. For solar metallicities and initial masses of $\leqslant 2.25 M_{\odot}$ LFB appears at effective temperatures lower than $4800 \mathrm{~K}$ (e.g., Charbonnel \& Balachandran 2000; Monaco et al. 2011).

As is seen in Figure 17, the evolved stars of our sample (thick+thin disk) are located approximately between $4800-5400 \mathrm{~K}$ in $T_{\text {eff }}$ and $2.2-2.8$ in $\log g$. The stars illustrated with filled circles have low ${ }^{12} \mathrm{C} /{ }^{13} \mathrm{C}$ values $(\leqslant 20)$ and they clearly reside far away from LFB. We suggest that the stars with especially low isotopic ratios, ${ }^{12} \mathrm{C} /{ }^{13} \mathrm{C} \leqslant 10$, may have evolved from lower initial masses and undergone a major extra-mixing processes when they passes the LFB. The amount of the extramixing is related to both the initial mass and the metallicity of the star, which may explain the deviation in the ${ }^{12} \mathrm{C} /{ }^{13} \mathrm{C}$ values $(\leqslant 20)$.

Stars with masses higher than $2.25 M_{\odot}$ ignite their central $\mathrm{He}$ before the core becomes degenerate. The H-burning shell which surrounds the He-burning core remains until the second dredgeup and never reaches the region of molecular discontinuity left 


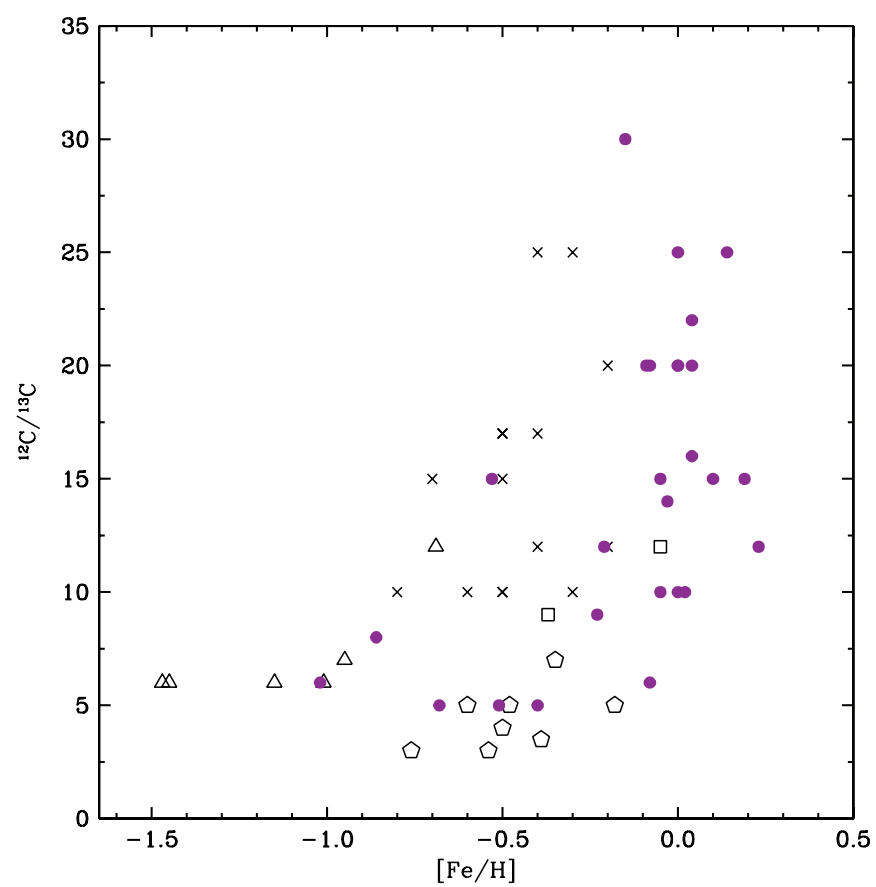

Figure 18. ${ }^{12} \mathrm{C} /{ }^{13} \mathrm{C}$ ratios (filled circles) as a function of the $[\mathrm{Fe} / \mathrm{H}]$ for $\mathrm{RHB}$, RC, and RC/RHB stars. Other RCs and RHBs from Lambert \& Ries (1981, open squares), Cottrell \& Sneden (1986, crosses), Gratton et al. (2000, triangles), and Tautvaišienè et al. (2001, open pentagons) are also plotted.

(A color version of this figure is available in the online journal.)

by the convective envelope during the first dredge-up phase. That is why stars with high initial masses are not expected to show the indicators of extra-mixing, such as low ${ }^{12} \mathrm{C} /{ }^{13} \mathrm{C}$ ratios (Charbonnel 1994; Charbonnel et al. 1998; Charbonnel \& Zahn 2007). In our sample, we have evolved stars with $20<$ ${ }^{12} \mathrm{C} /{ }^{13} \mathrm{C}<30$, which are close to the values suggested by canonical models (e.g., Schaller et al. 1992; Charbonnel 1994). These stars are shown with (blue) crosses in Figure 17. Assuming that these stars did have on average larger initial masses than the stars with low isotopic ratios, then the isotopic ratio issue is "solved," but leaves a problem: why are these stars now in the RHB domain instead of the RC? Here we suggest, but cannot prove, that the RHB stars with higher ${ }^{12} \mathrm{C} /{ }^{13} \mathrm{C}$ ratios have undergone substantial mass loss at some stage(s) of their evolution, leaving them with smaller present envelope masses. Such stars will appear bluer (hotter) than RC stars.

For further insight into Figure 18 we correlate ${ }^{12} \mathrm{C} /{ }^{13} \mathrm{C}$ ratios and $[\mathrm{Fe} / \mathrm{H}]$ metallicities. We include our $\mathrm{RHB}, \mathrm{RC}$, and $\mathrm{RC} / \mathrm{RHB}$ stars along with the data obtained by Lambert \& Ries (1981), Cottrell \& Sneden (1986), Gratton et al. (2000), and Tautvaišienè et al. (2001) for the same temperature, luminosity, and surface gravity range (same evolutionary stages). We have excluded SG stars and upper RGB stars in this plot. In Figure 18, we see a conspicuous trend with metallicity, similar to the one shown in Sneden (1991): lower metallicity RHB and RC stars have a smaller ${ }^{12} \mathrm{C} /{ }^{13} \mathrm{C}$ range (and lower values on average) than do similar objects of higher metallicity. As discussed in, e.g., Kalirai et al. (2009) and Catelan (2009), mass loss in evolved stars increases rapidly with increasing metallicity. If the ${ }^{12} \mathrm{C} /{ }^{13} \mathrm{C}$ ratio is also related to mass loss along with mixing processes then for a given metallicity, stars with different mass loss and mixing history must be responsible from the ${ }^{12} \mathrm{C} /{ }^{13} \mathrm{C}$ fluctuations seen toward higher metallicities in Figure 18.
According to Charbonnel (1994), the peak of the ${ }^{13} \mathrm{C}$ abundance in the inner region of a star is shifted outward toward high initial masses. If the star evolved from high masses $\left(>2.25 M_{\odot}\right)$, then different mechanisms, such as winds on the RGB, for mass loss should come into play other than He-flash. Depending on how massive the star is at the beginning, it might be possible for a star to dredge up some or all of its region, where the ${ }^{13} \mathrm{C}$ abundance peaks, during its RGB evolution. Since the thin-disk stars are more metal-rich compared to thick disk and halo stars, the thin-disk stars with higher metallicities or higher masses or a combination of both might have very low ${ }^{12} \mathrm{C} /{ }^{13} \mathrm{C}$ rates or not show any ${ }^{13} \mathrm{CN}$ future depending on the mass fraction lost during the RGB evolution. High-mass evolving stars appear to have more options to produce a variety of surface abundances. Figure 18 should become more illuminating if we increase the number of the evolved stars that are the members of the same population. If for example we can substantially augment the RHB thick- and thin-disk samples, then we might have a better understanding of the mechanisms alters the $\mathrm{CN}$ ratios during their post-MS evolution.

We conclude by re-emphasizing our basic result: the RHB $T_{\text {eff }}-\log g$ domain of the Galactic field is populated by subsolar-metallicity thick-disk stars (as expected) but also by high-metallicity thin-disk stars. We will be gathering more thin- and thick-disk data in order to substantially increase the RHB samples of different populations to try to set additional constraints on the mechanisms that may lead to stars ending up in this relatively rare evolutionary state.

We thank Bertrand Plez for sharing his $\mathrm{CH}$ line lists with us, and Andy McWilliam and Inese Ivans for making their model atmosphere interpolation software available to us. M.A. also thanks The Scientific and Technological Research Council of Turkey (TÜBITAK) for supporting her research by the "Postdoctoral Research Scholarship" program. This research has made use of NASA's Astrophysics Data System Bibliographic Services; the SIMBAD database and the VizieR service, both operated at CDS, Strasbourg, France; and the NASA/IPAC Extragalactic Database (NED) which is operated by the Jet Propulsion Laboratory, Caltech, under contract with NASA. Support for this study has come from the U.S. National Science Foundation grant AST 09-08978 and the Rex G. Baker, Jr. endowment to the University of Texas, Department of Astronomy.

\section{APPENDIX}

\section{NOTES ON SOME SELECTED STARS}

HIP 46325. Super metal-rich (SMR, $[\mathrm{Fe} / \mathrm{H}]>0.2)$ star. $[\mathrm{Fe} / \mathrm{H}]=0.46$. Possible member of a moving group HR 1614 (Feltzing \& Holmberg 2000). With $V_{\text {tot }}=64 \mathrm{~km} \mathrm{~s}^{-1}$, probable thick-disk member with high metallicity. Probable planet host (Robinson et al. 2007).

HIP 38801. $[\mathrm{Fe} / \mathrm{H}]=-0.06$. High-temperature star. It shows relatively high turbulence. It has peculiarly high La II and Eu II abundances. Has ${ }^{12} \mathrm{C}$ deficiency and ${ }^{14} \mathrm{~N}$ enhancement indicating a more evolved star than an $\mathrm{SG}$ but no ${ }^{12} \mathrm{C} /{ }^{13} \mathrm{C}$ ratio could be detected due to weak ${ }^{13} \mathrm{CH}$ or ${ }^{13} \mathrm{CN}$ features.

\section{REFERENCES}

Allende Prieto, C., Lambert, D. L., \& Asplund, M. 2001, ApJ, 556, L63 Alonso, A., Arribas, S., \& Martínez-Roger, C. 1999, A\&AS, 140, 261 Anthony-Twarog, B. J., Atwell, J., \& Twarog, B. A. 2005, AJ, 129, 872 Aoki, W., Honda, S., Beers, T. C., \& Sneden, C. 2003a, ApJ, 586, 506 
Aoki, W., Ryan, S. G., Iwamoto, N., et al. 2003b, ApJ, 592, L67

Asplund, M., Grevesse, N., Sauval, A. J., \& Scott, P. 2009, ARA\&A, 47, 481

Bensby, T., \& Feltzing, S. 2010, in IAU Symp. 265, Chemical Abundances in the Universe: Connecting First Stars to Planets, ed. K. Cunha, M. Spite, \& B. Barbuy (Cambridge: Cambridge Univ. Press), 300

Bensby, T., Feltzing, S., \& Lundström, I. 2003, A\&A, 410, 527

Bensby, T., Feltzing, S., \& Lundström, I. 2004, A\&A, 415, 155

Bertelli, G., Girardi, L., Marigo, P., \& Nasi, E. 2008, A\&A, 484, 815

Boothroyd, A. I., \& Sackmann, I. J. 1999, ApJ, 510, 232

Cannon, R. D. 1970, MNRAS, 150, 111

Castelli, F., Gratton, R. G., \& Kurucz, R. L. 1997, A\&A, 318, 841

Castelli, F., \& Kurucz, R. L. 2003, in IAU Symp. 210, Modelling of Stellar Atmospheres, ed. N. Piskunov, W. W. Weiss, \& D. F. Gray (Cambridge: Cambridge Univ. Press), 20

Catelan, M. 2009, in IAU Symp. 258, The Ages of Stars, ed. E. E. Mamajek, D R. Soderblom, \& R. F. G. Wyse (Cambridge: Cambridge Univ. Press), 209

Charbonnel, C. 1994, A\&A, 282, 811

Charbonnel, C., \& Balachandran, S. C. 2000, A\&A, 359, 563

Charbonnel, C., Brown, J. A., \& Wallerstein, G. 1998, A\&A, 332, 204

Charbonnel, C., \& Lagarde, N. 2010, A\&A, 522, 10

Charbonnel, C., \& Zahn, J.-P. 2007, A\&A, 467, L15

Chen, B., Vergely, J. L., Valette, B., \& Carraro, G. 1998, A\&A, 336, 137

Chen, Y. Q., Nissen, P. E., Zhao, G., Zhang, H. W., \& Benoni, T. 2000, A\&AS, 141,491

Cottrell, P. L., \& Sneden, C. 1986, A\&A, 161, 314

Dehnen, W., \& Binney, J. J. 1998, MNRAS, 298, 387

Delbouille, L., Roland, G., \& Neven, L. 1973, Atlas photometrique du spectre solaire de $\lambda 3000$ a $\lambda 10000$ (Liege: Universite de Liege, Institut d'Astrophysique)

Dinescu, D. I., Girard, T. M., \& van Altena, W. F. 1999, AJ, 117, 1792

Feltzing, S., \& Holmberg, J. 2000, A\&A, 357, 153

Fitzpatrick, M. J. 1993, in ASP Conf. Ser. 52, Astronomical Data Analysis Software and Systems II, ed. R. J. Hanisch, R. V. J. Brissenden, \& J. Barnes (San Francisco, CA: ASP), 472

Fitzpatrick, M. J., \& Sneden, C. 1987, BAAS, 19, 1129

Franchini, M., Morossi, C., Di Marcantonio, P., et al. 2004, ApJ, 613, 312

Gilmore, G., \& Reid, N. 1983, MNRAS, 202, 1025

Gratton, R. G., Carretta, E., Eriksson, K., \& Gustafsson, B. 1999, A\&A, 350, 955

Gratton, R. G., Sneden, C., Carretta, E., \& Bragaglia, A. 2000, A\&A, 354, 169

Gray, D. F., \& Brown, K. 2001, PASP, 113, 723

Gray, R. O., \& Corbally, C. J. 2009, Stellar Spectral Classification (Princeton, NJ: Princeton Univ. Press)

Grevesse, N., \& Sauval, A. J. 1998, Space Sci. Rev., 85, 161

Griffin, R. 1968, A Photometric Atlas of the Spectrum of Arcturus, $\lambda \lambda 3600-8825 \AA$ (Cambridge: Cambridge Philosophical Society)

Hakkila, J., Myers, J. M., Stidham, B. J., \& Hartmann, D. H. 1997, AJ, 114 , 2043

Harlan, E. A. 1969, AJ, 74, 916

Harlan, E. A. 1974, AJ, 79, 682

Harlan, E. A. 1981, AJ, 86, 1896

Henry, G. W., Fekel, F. C., Henry, S. M., \& Hall, D. S. 2000, ApJS, 130, 201

Hesser, J. E., Harris, W. E., Vandenberg, D. A., et al. 1987, PASP, 99, 739

Hill, V., Plez, B., Cayrel, R., et al. 2002, A\&A, 387, 560

Hollek, J. K., Frebel, A., Roederer, I. U., et al. 2011, ApJ, 742, 54

Iben, I., Jr. 1964, ApJ, 140, 1631

Iben, I., Jr. 1965, ApJ, 142, 1447

Iben, I., Jr. 1967a, ApJ, 147, 624

Iben, I., Jr. 1967b, ApJ, 147, 650

Ivans, I. I., Simmerer, J., Sneden, C., et al. 2006, ApJ, 645, 613

Jacobson, H. R., Friel, E. D., \& Pilachowski, C. A. 2011, AJ, 141, 58

Johansson, S., Litzén, U., Lundberg, H., \& Zhang, Z. 2003, ApJ, 584, L107

Johnson, D. R. H., \& Soderblom, D. R. 1987, AJ, 93, 864

Kaempf, T. A., de Boer, K. S., \& Altmann, M. 2005, A\&A, 432, 879

Kalirai, J. S., Saul Davis, D., Richer, H. B., et al. 2009, ApJ, 705, 408

Kjaergaard, P., Gustafsson, B., Walker, G. A. H., \& Hultqvist, L. 1982, A\&A, 115,145

Kumar, Y. B., Reddy, B. E., \& Lambert, D. L. 2011, ApJ, 730, L12

Kurucz, R. L. 2011, Can. J. Phys., 89, 417

Lallement, R., Welsh, B. Y., Vergely, J. L., Crifo, F., \& Sfeir, D. 2003, A\&A, 411,447

Lambert, D. L., \& Ries, L. M. 1981, ApJ, 248, 228

Lawler, J. E., Bonvallet, G., \& Sneden, C. 2001a, ApJ, 556, 452
Lawler, J. E., Wickliffe, M. E., den Hartog, E. A., \& Sneden, C. 2001b, ApJ, 563, 1075

Lee, Y. S., Beers, T. C., An, D., et al. 2011, ApJ, 738, 187

Lee, Y.-W., Demarque, P., \& Zinn, R. 1994, ApJ, 423, 248

Liu, Y., Sato, B., Takeda, Y., Ando, H., \& Zhao, G. 2010, PASJ, 62, 1071

Luck, R. E., \& Heiter, U. 2007, AJ, 133, 2464

Mallik, S. V. 1998, A\&A, 338, 623

Mashonkina, L., Gehren, T., Shi, J.-R., Korn, A. J., \& Grupp, F. 2011, A\&A, 528, A87

McWilliam, A. 1990, ApJS, 74, 1075

Mishenina, T. V., Bienaymé, O., Gorbaneva, T. I., et al. 2006, A\&A, 456, 1109

Mishenina, T. V., Gorbaneva, T. I., Bienaymé, O., et al. 2007, Astron. Rep., 51 382

Monaco, L., Villanova, S., Moni Bidin, C., Carraro, G., et al. 2011, A\&A, 529, A90

Moore, C. E., Minnaert, M. G. J., \& Houtgast, J. 1966, The Solar Spectrum $2935 \AA$ to $8770 \AA$ (National Bureau of Standards Monograph; Washington, DC: US Government Printing Office)

Nissen, P. E. 2004, in Origin and Evolution of the Elements, Carnegie Observatories Astrophysics Series, Vol. 4, ed. A. McWilliam \& M. Rauch (Pasadena, CA: Carnegie Observatories), 154

Nordström, B., Mayor, M., Andersen, J., et al. 2004, A\&A, 418, 989

Norris, J. 1987, AJ, 93, 616

Omiya, M., Izumiura, H., Han, I., et al. 2009, PASJ, 61, 825

Perryman, M. A. C., Lindegren, L., Kovalevsky, J., et al. 1997, A\&A, 323, L49

Piersanti, L., Tornambé, A., \& Castellani, V. 2004, MNRAS, 353, 243

Plez, B., \& Cohen, J. G. 2005, A\&A, 434, 1117

Ralchenko, Yu., Kramida, A. E., Reader, J., \& NIST ASD Team 2011, NIST Atomic Spectra Database, ver. 4.1.0 (Gaithersburg, MD: National Institute of Standards and Technology)

Ramírez, I., \& Meléndez, J. 2005, ApJ, 626, 465

Reddy, B. E., Lambert, D. L., \& Allende Prieto, C. 2006, MNRAS, 367, 1329

Reddy, B. E., Tomkin, J., Lambert, D. L., \& Allende Prieto, C. 2003, MNRAS, 340,304

Robinson, S. E., Ammons, S. M., Kretke, K. A., et al. 2007, ApJS, 169, 430

Roederer, I. U., Lawler, J. E., Sneden, C., et al. 2008, ApJ, 675, 723

Roederer, I. U., Marino, A. F., \& Sneden, C. 2011, ApJ, 742, 37

Rose, J. A. 1985, AJ, 90, 787

Rosenberg, A., Aparicio, A., Saviane, I., \& Piotto, G. 2000a, A\&AS, 145, 451

Rosenberg, A., Piotto, G., Saviane, I., \& Aparicio, A. 2000b, A\&AS, 144, 5

Ryan, S. G., \& Lambert, D. L. 1995, AJ, 109, 2068

Sandage, A. 1972, ApJ, 178, 1

Schaller, G., Schaerer, D., Meynet, G., \& Maeder, A. 1992, A\&AS, 96, 269

Simmerer, J., Sneden, C., Cowan, J. J., et al. 2004, ApJ, 617, 1091

Smith, V. V., Lambert, D. L., \& Nissen, P. E. 1998, ApJ, 506, 405

Sneden, C. 1973, ApJ, 184, 839

Sneden, C. 1991, in IAU Symp. 145, Evolution of Stars: The Photospheric Abundance Connection, ed. G. Michaud \& A. V. Tutukov (Dordrecht: Kluwer), 235

Sneden, C., Cowan, J. J., \& Gallino, R. 2008, ARA\&A, 46, 241

Sneden, C., Cowan, J. J., Lawler, J. E., et al. 2002, ApJ, 566, L25

Sneden, C., Pilachowski, C. A., \& VandenBerg, D. A. 1986, ApJ, 311, 826

Soubiran, C., Bienaymé, O., Mishenina, T. V., \& Kovtyukh, V. V. 2008, A\&A, 480,91

Soubiran, C., \& Girard, P. 2005, A\&A, 438, 139

Stetson, P. B., \& Aikman, C. L. 1987, AJ, 93, 1439

Straižys, V., Bartkevicius, A., \& Sperauskas, J. 1981, A\&A, 99, 152

Strassmeier, K. G., \& Schordan, P. 2000, Astron. Nachr., 321, 277

Sweigart, A. V., \& Gross, P. G. 1978, ApJS, 36, 405

Takeda, Y., Sato, B., Kambe, E., et al. 2005, PASJ, 57, 109

Takeda, Y., Sato, B., \& Murata, D. 2008, PASJ, 60, 781

Tautvaišienè, G. 1996, Astron. Nachr., 317, 29

Tautvaišienė, G. 1997, MNRAS, 286, 948

Tautvaišienè, G., Edvardsson, B., Tuominen, I., \& Ilyin, I. 2001, A\&A, 380, 578

Thorén, P., Edvardsson, B., \& Gustafsson, B. 2004, A\&A, 425, 187

Tull, R. G., MacQueen, P. J., Sneden, C., \& Lambert, D. L. 1995, PASP, 107, 251

Upgren, A. R. 1962, AJ, 67, 37

Upgren, A. R. 1963, AJ, 68, 194

van Leeuwen, F. 2007, A\&A, 474, 653

Venn, K. A., Irwin, M., Shetrone, M. D., et al. 2004, AJ, 128, 1177

Wilson, O. C. 1976, ApJ, 205, 823

Zahn, J.-P. 1992, A\&A, 265, 115 